\title{
Rapid Access to Pyrazolo[3,4-c]pyridines via Alkyne Annulation: Limitations of Steric Control in Nickel Catalyzed Alkyne Insertions
}

\author{
Stephen T. Heller ${ }^{* a}$ and Swaminathan R. Natarajan ${ }^{b}$ \\ ${ }^{a}$ Department of Chemistry, University of California, Berkeley, California 94720 \\ ${ }^{b}$ Department of Medicinal Chemistry, Merck Research Laboratories, P.O. Box 2000, Rahway, New Jersey \\ 07065 \\ stheller@berkeley.edu \\ Supporting Information
}


Table of Contents

\begin{tabular}{|c|c|}
\hline Item & Page \\
\hline General Comments & 3 \\
\hline 1 & 4 \\
\hline Procedure for Preparation of 1-alkyl-dimethoxymethyl-(1H)-pyrazoles & 4 \\
\hline 3a & 5 \\
\hline 3b & 5 \\
\hline Representative Procedure for Iodination of Pyrazoles & 5 \\
\hline Representative Procedure for Hydrolysis of Dimethoxymethyl- $(1 H)$-pyrazoles & 6 \\
\hline $4 a$ & 6 \\
\hline 4b & 7 \\
\hline Representative Procedure for Nickel Catalyzed Annulation & 7 \\
\hline 5 & 7 \\
\hline 6 & 8 \\
\hline 7 & 9 \\
\hline 8a & 9 \\
\hline 8b & 9 \\
\hline 9 & 10 \\
\hline 10a & 10 \\
\hline 10b & 10 \\
\hline 11a & 11 \\
\hline 12 & 11 \\
\hline 13 & 12 \\
\hline 14 & 12 \\
\hline 15 & 12 \\
\hline $16 \mathbf{a}$ & 13 \\
\hline $16 b$ & 13 \\
\hline 17 & 13 \\
\hline 18 & 14 \\
\hline 19 & 14 \\
\hline${ }^{1} H$ NMR spectra: & $15-59$ odds \\
\hline 1 & 15 \\
\hline 3a & 17 \\
\hline $3 \mathbf{b}$ & 19 \\
\hline $4 a$ & 21 \\
\hline $4 b$ & 23 \\
\hline 5 & 25 \\
\hline 6 & 27 \\
\hline 7 & 29 \\
\hline $8 a$ & 31 \\
\hline $8 \mathbf{b}$ & 33 \\
\hline 9 & 35 \\
\hline 10a & 37 \\
\hline $10 \mathrm{~b}$ & 39 \\
\hline 11a & 41 \\
\hline
\end{tabular}




\begin{tabular}{|c|c|c|}
\hline & 12 & 43 \\
\hline & 13 & 45 \\
\hline & 14 & 47 \\
\hline & 15 & 49 \\
\hline & $16 a$ & 51 \\
\hline & $16 b$ & 53 \\
\hline & 17 & 55 \\
\hline & 18 & 57 \\
\hline & 19 & 59 \\
\hline \multirow[t]{24}{*}{${ }^{13} \mathrm{C} N M R$ spectra } & & $16-60$ evens \\
\hline & 1 & 16 \\
\hline & $3 a$ & 18 \\
\hline & $3 \mathbf{b}$ & 20 \\
\hline & $4 a$ & 22 \\
\hline & $4 b$ & 24 \\
\hline & 5 & 26 \\
\hline & 6 & 28 \\
\hline & 7 & 30 \\
\hline & $8 a$ & 32 \\
\hline & $8 \mathbf{b}$ & 34 \\
\hline & 9 & 36 \\
\hline & $10 a$ & 38 \\
\hline & $10 \mathrm{~b}$ & 40 \\
\hline & 11a & 42 \\
\hline & 12 & 44 \\
\hline & 13 & 46 \\
\hline & 14 & 48 \\
\hline & 15 & 50 \\
\hline & $16 a$ & 52 \\
\hline & $16 b$ & 54 \\
\hline & 17 & 56 \\
\hline & 18 & 58 \\
\hline & 19 & 60 \\
\hline
\end{tabular}

\section{General Comments}

All chemicals were purchased from Aldrich Chemical Company and were used without further purification unless otherwise noted. Anhydrous solvents were purchased from Acros and were stored over activated $4 \AA$ molecular sieves. NMR spectra were taken with a Varian Inova (400 MHz, $500 \mathrm{MHz}$, or $600 \mathrm{MHz}$ ) spectrometer. LC/MS were obtained using an Agilent 1100 series LC and Micromass Zq spectrometer. Column 
chromatography was performed using a Biotage Horizon system utilizing standard prepacked silica gel cartridges.

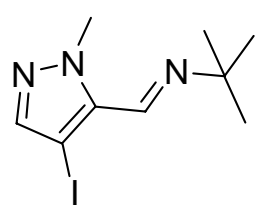

tert-butyl 3-(4-chlorophenyl)-4-iodo-1-methyl-(1H)-pyrazole-5-carboximine (1). 4-iodo-1-methyl-(1H)-pyrazole (4.0 g, 19.23 mmol, Boron Molecular Inc.) was dissolved in $100 \mathrm{~mL}$ dry $\mathrm{THF}$ and the resulting solution was cooled to $-20^{\circ} \mathrm{C}$. Freshly prepared lithium diisopropylamide (21 mmol, $1.0 \underline{\mathrm{M}}$ ) was added dropwise and the resulting orange solution was stirred for 2 hours at $-20^{\circ} \mathrm{C}$, at which time DMF $(4.47 \mathrm{~mL}, 57.7 \mathrm{mmol})$ was added in one portion and the mixture was allowed to warm to room temperature overnight. The reaction mixture was quenched with saturated $\mathrm{NH}_{4} \mathrm{Cl}$ solution at room temperature and the organic layer was collected. The aqueous layer was extracted twice with $\mathrm{Et}_{2} \mathrm{O}$ and then the pooled organics were washed with $1 \mathrm{~N} \mathrm{HCl}(100 \mathrm{~mL})$, water, and brine. The organic fraction was dried over sodium sulfate and concentrated in vacuo to afford an orange solid that was purified by column chromatography (1:4 ethyl acetate : hexanes) to afford 4-iodo-1-methyl-5-(1H)-pyrazolecarboxaldehyde (2.50 g, 55\%).

${ }^{1}$ H NMR (500 MHz, $\left.\mathrm{CDCl}_{3}\right) \delta$ 9.80, (s, 1H), 7.57 (s, 1H), 4.18 (s, 3H).

The above aldehyde $(2.0 \mathrm{~g}, 8.47 \mathrm{mmol})$ was dissolved in tert-butylamine (10 mL, $6.96 \mathrm{~g}$, $95 \mathrm{mmol}$ ) in a pressure tube and the mixture was heated to $100^{\circ} \mathrm{C}$ overnight. The reaction was cooled to room temperature and the resulting solution was diluted with ether $(100 \mathrm{~mL})$. The solution was then dried with sodium sulfate and concentrated in vacuo to afford 1 as a white solid (2.37 g, 96\%). ${ }^{1} \mathbf{H}$ NMR (600 MHz, $\left.\mathrm{CDCl}_{3}\right) \delta 8.20$ (s, 1H), 7.48 (s, 1H), 4.19 (s, 3H), 1.29 (s, 9H); ${ }^{13} \mathrm{C}$ NMR (600 MHz, $\left.\mathrm{CDCl}_{3}\right) \delta 145.1,143.0,136.8$, 63.4, 58.3, 40.1, 29.4

\section{Procedure for Preparation of 1-alkyl-dimethoxymethyl-(1H)-pyrazoles:}

Pyruvic aldehyde dimethyl acetal $(0.474 \mathrm{~mL}, 0.474 \mathrm{~g}, 4 \mathrm{mmol})$ was dissolved in $10 \mathrm{~mL}$ dry toluene in a $50 \mathrm{~mL}$ single neck round bottom flask and the solution was cooled to $0^{\circ} \mathrm{C}$. The solution was stirred vigorously and LiHMDS (4.2 mL, 1.0 $\underline{\mathrm{M}}$ in THF) was 
added rapidly in one portion. The resulting bright yellow solution was allowed to stir for 1 minute and then 4-chlorobenzoyl chloride $(0.350 \mathrm{~g} 0.256 \mathrm{~mL}, 2 \mathrm{mmol})$ was added in one portion rapidly. The reaction was removed from the ice bath and allowed to stir for 5 minutes, at which time acetic acid $(0.126 \mathrm{~mL}, 0.132 \mathrm{~g}, 2.2 \mathrm{mmol})$ in $10 \mathrm{~mL}$ methanol was added. The flask was placed back in the ice bath and methylhydrazine (0.194 g, 4.2 mmol) was added. The mixture was then brought to reflux for 15-20 minutes. $20 \mathrm{~mL}$ saturated $\mathrm{NaHCO}_{3}$ was added and the mixture was extracted twice with ethyl acetate. The pooled organics were washed with brine, dried over sodium sulfate, and concentrated in vacuo to afford product which was purified by column chromatography to afford:

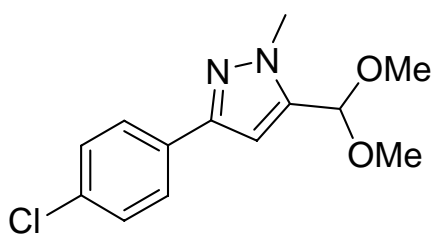

\section{3-(4-chlorophenyl)-5-dimethoxymethyl-1-methyl-(1H)-pyrazole (3a).}

Column chromatography (1:3 ethyl acetate:hexanes) yielded a colorless oil (0.230 g, 43\%). ${ }^{1} \mathbf{H}$ NMR (400 MHz, $\left.\mathrm{CDCl}_{3}\right) \delta 7.71$ (d, 2H, J = 8.4 Hz), 7.34 (d, 2H, J = 8.4 Hz), 6.61 (s, 1H), 5.49 (s, 1H), 3.91 (s, 3H), 3.36 (s, 6H); $\left.{ }^{13} \mathbf{C ~ N M R ~ ( 6 0 0 ~ M H z , ~} \mathrm{CDCl}_{3}\right) \delta$ 148.4, 140.3, 133.0, 131.8, 128.5, 126.4, 103.3, 97.0, 52.6, 37.2;

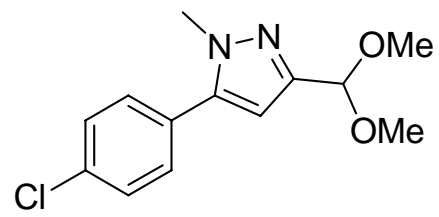

\section{5-(4-chlorophenyl)-3-dimethoxymethyl-1-methyl-(1H)-pyrazole (3b).}

Column chromatography (1:2 ethyl acetate:hexanes) yielded a colorless oil (0.143 g, 27\%). ${ }^{1} \mathbf{H}$ NMR (600 MHz, $\left.\mathrm{CDCl}_{3}\right) \delta 7.43$ (d, 2H, J = 8.4 Hz), 7.35 (d, 2H, J = 8.4 Hz), 6.37 (s, 1H), 5.49 (s, 1H), 3.87 (s, 3H), 3.43 (s, 6H); $\left.{ }^{13} \mathbf{C ~ N M R ~ ( 6 0 0 ~ M H z , ~} \mathrm{CDCl}_{3}\right) \delta$ 149.0, 143.0, 135.5, 134.5, 129.8, 128.8, 128.7, 104.2, 99.6, 53.0, 37.4

\section{Representative Procedure for Iodination of Pyrazoles:}

3-(4-chlorophenyl)-5-dimethoxymethyl-1-methyl-(1H)-pyrazole (0.643 g, $2.41 \mathrm{mmol})$ was dissolved in $25 \mathrm{~mL}$ dichloromethane and powdered potassium carbonate $(1.0 \mathrm{~g}, 7.23$ 
mmol) was added. The mixture was stirred at room temperature for 5 minutes, at which time solid iodine monochloride (1.173 g, $7.23 \mathrm{mmol})$ which had been dissolved in $15 \mathrm{~mL}$ dichloromethane was added dropwise at $0^{\circ} \mathrm{C}$. The resulting mixture was stirred for 20 hrs at room temperature. After the allotted reaction time, the mixture was carefully quenched with $2 \mathrm{~N}$ sodium thiosulfate solution and the mixture was vigorously stirred until no purple color remained. The biphasic mixture diluted with $20 \mathrm{~mL}$ dichloromethane and $20 \mathrm{~mL}$ water and the organic fraction was separated, dried over sodium sulfate, and concentrated in vacuo to afford 3-(4-chlorophenyl)-5dimethoxymethyl-4-iodo-1-methyl-(1H)-pyrazole as a white solid. This material was used as-is in the hydrolysis step but yields of 74-81\% were generally achieved.

\section{Representative Procedure for Hydrolysis of Dimethoxymethyl-(1H)-pyrazoles:}

3-(4-chlorophenyl)-5-dimethoxymethyl-4-iodo-1-methyl-(1H)-pyrazole from the previous step was dissolved in $10 \mathrm{~mL}$ acetic acid and $10 \mathrm{~mL}$ water. The mixture was then stirred for $1 \mathrm{hr}$ at room temperature. The mixture was then diluted with $20 \mathrm{~mL}$ water and extracted with dichloromethane (3x). The organic fraction was dried over sodium sulfate and concentrated in vacuo to afford 3-(4-chlorophenyl)-4-iodo-1-methyl-(1H)-5pyrazolecarboxaldehyde, the resulting material was used directly in imine formation (7080\% yield over two steps). NOTE: Insoluble substrates should be dissolved by adding a minimal amount of THF to the acetic acid/water solution.

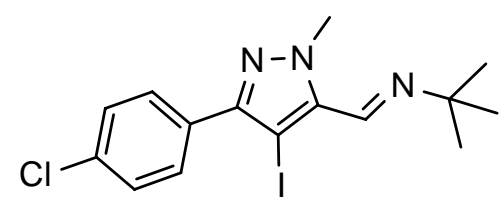

tert-butyl 3-(4-chlorophenyl)-4-iodo-1-methyl-(1H)-pyrazolo[3,4-c]pyridine-5carboximine (4a).

3-(4-chlorophenyl)-4-iodo-1-methyl-5-(1H)-5-pyrazolecarboxaldehyde (0.863 g, 2.5 mmol) was reacted with tert-butylamine in analogous fashion to $\mathbf{1}$ to afford $\mathbf{4 a}$ as a cream colored solid (0.980 g, 99\%). ${ }^{1} \mathbf{H}$ NMR (400 MHz, CDCl $) \delta 8.28$ (s, 1H), 7.76 (d 2H, J = 8.4 Hz), 7.41 (d, 2H, J = 8.4 Hz), 4.23 (s, 2H), 1.32 (s, 9H); ${ }^{13}$ C NMR (600 MHz, $\left.\mathrm{CDCl}_{3}\right) \delta 146.8,146.1,138.5,134.2,131.2,129.4,128.5,63.7,58.6,40.4,29.5$ 


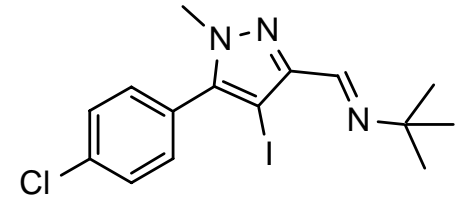

tert-butyl 5-(4-chlorophenyl)-4-iodo-1-methyl-(1H)-pyrazolo[3,4-c]pyridine-3carboximine (4b).

Prepared in analogous fashion to $\mathbf{4 a}$. Obtained as a white powder, (0.553, 97\%). ${ }^{1} \mathbf{H}$ NMR (400 MHz, $\left.\mathrm{CDCl}_{3}\right) \delta 8.30$ (s, 1H), 7.50 (d, 2H, $J=8.4 \mathrm{~Hz}$ ), 7.31 (d, 2H, $J=8.4$ Hz), 3.87 (s, 3H), 1.35 (s, 9H); ${ }^{13}$ C NMR (600 MHz, $\left.\mathrm{CDCl}_{3}\right) \delta$ 148.7, 147.9, 136.0, 131.6, 129.4, 127.9, $63.658 .3,38.9,29.9$

\section{Representative Procedure for Nickel Catalyzed Annulation:}

Powdered zinc (0.090 g, $1.374 \mathrm{mmol})$ and $\mathrm{Ni}\left(\mathrm{PPh}_{3}\right)_{2} \mathrm{Br}_{2}$ (0.026 g, $0.034 \mathrm{mmol}, 5 \mathrm{~mol} \%$, Strem Chemicals, Inc.), were added to a flask that was subsequently degassed. $10 \mathrm{~mL}$ dry acetonitrile was then added, followed by 1 ( $0.2 \mathrm{~g}, 0.687 \mathrm{mmol})$, and then diphenylacetylene ( $0.122 \mathrm{~g}, 0.687 \mathrm{mmol})$. The mixture was carefully degassed once more and then heated to $80^{\circ} \mathrm{C}$ for two hours, at which time the reaction mixture was colorless. The mixture was cooled to room temperature, diluted with $10 \mathrm{~mL}$ acetonitrile, and filtered through Celite. The filtrate was concentrated in vacuo to afford a yellow resin that was purified as described below:

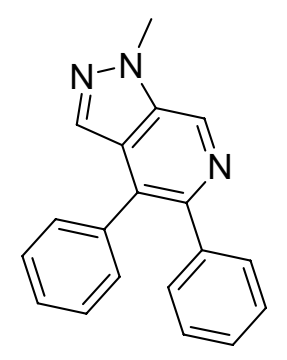

\section{4,5-diphenyl-1-methyl-(1H)-pyrazolo[3,4-c]pyridine (5).}

Purified by column chromatography (1:1 ethyl acetate:hexanes) to yield a white solid (0.161 g, 82\%). ${ }^{1} \mathbf{H}$ NMR (600 MHz, $\left.\mathrm{CDCl}_{3}\right) \delta 9.01$ (s, 1H), 7.92 (s, 1H), 7.38-7.6 (m, 2H), 7.34-7.30 (m, 5H), 7.24-7.21 (m, 3H) 4.23 (s, 3H); $\left.{ }^{13} \mathbf{C ~ N M R ~ ( 6 0 0 ~ M H z , ~} \mathrm{CDCl}_{3}\right) \delta$ 
146.4, 140.1, 137.1, 135.9, 132.7, 131.9, 130.3, 130.1, 129.4, 128.4, 127.8, 127.5, 127.0, 36.2 (one peak overlaps); MS calcd for $\mathrm{C}_{19} \mathrm{H}_{16} \mathrm{~N}_{3}[\mathrm{M}+\mathrm{H}]^{+}$, 286.13; found, 286.17.

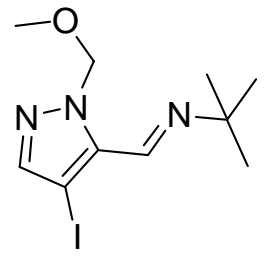

tert-butyl 3-(4-chlorophenyl)-4-iodo-1-(methoxymethyl)-(1H)-pyrazole-5carboximine (6).

Sodium hydride (0.928 g, $23.2 \mathrm{mmol}, 60 \% \mathrm{wt}$ in mineral oil) was suspended in $25 \mathrm{~mL}$ dry THF and stirred for 15 minutes. Then a solution of 4-iodopyrazole (3.0 g, 15.47 $\mathrm{mmol}$ ) in $25 \mathrm{~mL}$ dry THF was added dropwise at $0^{\circ} \mathrm{C}$. When the addition was finished the mixture was stirred at $40^{\circ} \mathrm{C}$ for $1 \mathrm{hr}$. Chloromethyl methyl ether (1.292 mL, 17.01 mmol) was added in one portion and the mixture was allowed to stir at $50^{\circ} \mathrm{C}$ for 4 hrs and then room temperature overnight. The mixture was quenched with sat. $\mathrm{NaHCO}_{3}$ and extracted with ethyl acetate (3x). The organic fractions were washed with brine, dried over sodium sulfate and concentrated in vacuo to afford 4-iodo-1-(methoxymethyl)pyrazole as a yellow oil (3.57 g, 97\%) ${ }^{1} \mathbf{H}$ NMR (500 MHz, $\left.\mathrm{CDCl}_{3}\right) \delta 7.63(\mathrm{~s}, 1 \mathrm{H}), 7.58$ (s, 1H), 5.39 (s, 2H), 3.34 (s, 3H) 1-(methoxymethyl)-4-iodopyrazole was dissolved in $100 \mathrm{~mL}$ dry THF and the solution was cooled to $-20^{\circ} \mathrm{C}$. Freshly prepared LDA (7.38, $14.77 \mathrm{mmol}$ ) was then added dropwise and the mixture was stirred at $-20^{\circ} \mathrm{C}$ for $2 \mathrm{hrs}$. DMF (4.57 mL, $59.1 \mathrm{mmol}$ ) was added in one portion. The solution was allowed to warm to room temperature over 4 hrs. Water was added and the mixture was extracted with ether (3x). The organic fractions were pooled, washed with brine, dried over sodium sulfate, and then concentrated in vacuo to afford a yellow resin. Silica gel chromatography (2:1 hexanes : ethyl acetate) afforded 4-iodo-1-(methoxymethyl)-5pyrazolecarboxaldehyde as an off-white solid (2.1 g, 54\%).

${ }^{1}$ H NMR (600 MHz, $\mathrm{CDCl}_{3}$ ) $\delta 9.84$ (s, 1H), 7.67 (s, 1H), 5.76 (s, 2H), 3.35 (s, 3H) 4-iodo-1-(methoxymethyl)-5-pyrazolecarboxaldehyde (0.3 g, $1.132 \mathrm{mmol}$ ) was dissolved in tert-butylamine $(1.2 \mathrm{~mL}, 11.32 \mathrm{mmol})$ in a sealed tube and was then heated to $100^{\circ} \mathrm{C}$ overnight. The solution was diluted with $20 \mathrm{~mL}$ ether and dried over sodium sulfate. The filtrate was concentrated in vacuo to afford 6 (0.352 g, 97\%) as a viscous yellow oil. 
${ }^{1}$ H NMR (600 MHz, CDCl ${ }_{3}$ ) $\delta 8.23$ (s, 1H), 7.58 (s, 1H), 5.94 (s, 2H), 3.35 (s, 3H), 1.30 (s, 9H); ${ }^{13}$ C NMR (600 MHz, $\left.\mathrm{CDCl}_{3}\right) \delta$ 144.7, 144.6, 137.6, 80.3, 65.7, 58.5, 56.6, 29.2

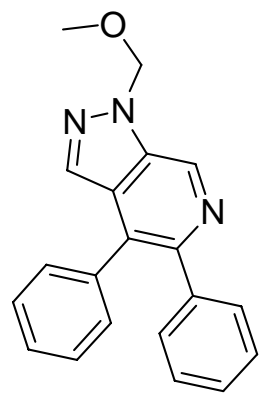

\section{4,5-diphenyl-1-(methoxymethyl)-(1H)-pyrazolo[3,4-c]pyridine (7).}

Purified by column chromatography (1:1 ethyl acetate:hexanes) to yield a white solid (0.1582 g, 77\%). ${ }^{1}$ H NMR (600 MHz, $\left.\mathrm{CDCl}_{3}\right) \delta 9.16$ (s, 1H), 7.98 (s, 1H), 7.39-7.31 (m, 7H), 7.24-7.22 (m, 3H), 5.83 (s, 2H), 3.38 (s, 3H); ${ }^{13} \mathbf{C}$ NMR (600 MHz, $\mathrm{CDCl}_{3}$ ) $\delta$ 147.5, 139.9, 136.9 135.6, 134.0, 132.4, 130.5, 130.3, 130.1, 128.5, 127.8, 127.6, 127.2, 127.1, 80.1, 56.8; MS calcd for $\mathrm{C}_{20} \mathrm{H}_{18} \mathrm{~N}_{3} \mathrm{O}[\mathrm{M}+\mathrm{H}]^{+}$, 316.14; found, 316.17

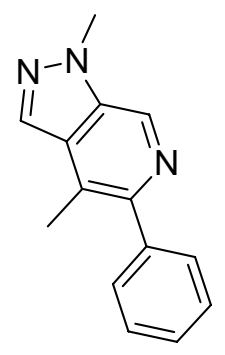

\section{1,4-dimethyl-5-phenyl-(1H)-pyrazolo[3,4-c]pyridine (8a).}

Purified by column chromatography (1:9 acetone:DCM) to yield a white solid (0.0603 g, 39\%). ${ }^{1} \mathbf{H}$ NMR (600 MHz, $\mathrm{CDCl}_{3}$ ) $\delta 8.87$ (s, $1 \mathrm{H}$ ), 8.07 (s, 1H), 7.55 (d, 2H, $J=6.6 \mathrm{~Hz}$ ), 7.45 (t, 2H), 7.38 (t, 1H), 4.20 (s, 3H), 2.60 (s, 3H); $\left.{ }^{13} \mathbf{C ~ N M R ~ ( 6 0 0 ~ M H z , ~} \mathrm{CDCl}_{3}\right) \delta$ 147.6, 140.4, 135.6, 131.7, 130.5, 129.9, 129.6, 128.1, 127.3, 122.0, 36.1, 16.2 ; MS calcd for $\mathrm{C}_{14} \mathrm{H}_{14} \mathrm{~N}_{3}[\mathrm{M}+\mathrm{H}]^{+}$, 224.12; found, 224.17

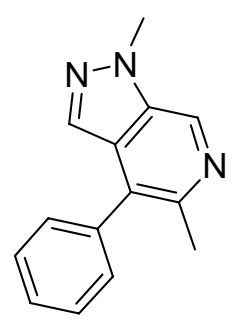

1,5-dimethyl-4-phenyl-(1H)-pyrazolo[3,4-c]pyridine (8b). 
Purified by column chromatography (1:4 acetone:DCM) to yield an off-white solid (0.0526 g, 34\%). ${ }^{1}$ H NMR (600 MHz, CDCl $) \delta 8.87$ (s, 1H), 7.74 (s, 1H), 7.67-7.65 (m, 2H), 7.53-7.50 (m, 1H), 7.43 (m, 2H), 4.19 (s, 3H), 2.59 (s, 3H); ${ }^{13}$ C NMR (600 MHz, $\left.\mathrm{CDCl}_{3}\right) \delta$ 144.1, 137.2, 135.6, 133.6, 131.8, 131.4, 130.0, 129.5, 128.6, 127.8, 36.2, 21.7; MS calcd for $\mathrm{C}_{14} \mathrm{H}_{14} \mathrm{~N}_{3}[\mathrm{M}+\mathrm{H}]^{+}$, 224.12; found, 224.17

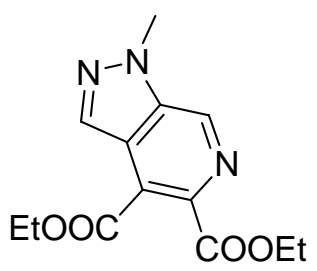

diethyl 3-methyl-(1H)-pyrazolo[3,4-c]pyridine-4,5-dicarboxylate (9).

Purified by column chromatography (1:1 ethyl acetate:hexanes) to yield a white solid (0.065 g, 34\%) ${ }^{1} \mathbf{H}$ NMR (600 MHz, $\mathrm{CDCl}_{3}$ ) $\delta 9.04$ (s, 1H), 8.31 (s, 1H), 4.52 (quartet, 2H), 4.49 (quartet, 2H), 1.46 (t, 3H), 1.44 (t, 3H); ${ }^{13} \mathbf{C}$ NMR (600 MHz, $\mathrm{CDCl}_{3}$ ) $\delta 166.4$, 165.2, 140.1, 136.7, 135.0, 133.5, 126.2, 120.0, 62.1, 36.4, 14.1; MS calcd for $\mathrm{C}_{13} \mathrm{H}_{16} \mathrm{~N}_{3} \mathrm{O}_{4}[\mathrm{M}+\mathrm{H}]^{+}$, 278.11; found, 278.16

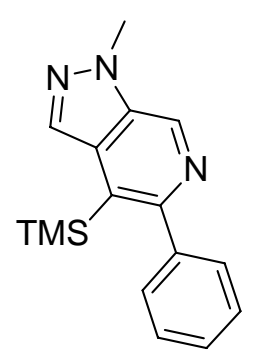

\section{5-phenyl-1-methyl-4-(trimethylsilyl)-(1H)-pyrazolo[3,4-c]pyridine (10a).}

Purified by column chromatography (1:1 ethyl acetate:hexanes) to yield a white solid (0.0398 g, 21\%) ${ }^{1}$ H NMR (600 MHz, $\left.\mathrm{CDCl}_{3}\right) \delta 8.94$ (s, 1H), 8.16 (s, 1H), 7.42-7.39 (m, 5H), 4.19 (s, 3H), 0.13 (s, 9H); ${ }^{13}$ C NMR (600 MHz, $\left.\mathrm{CDCl}_{3}\right) \delta$ 155.6, 143.9, 134.3, 133.7, 133.2, 132.7, 129.7, 127.8, 127.6, 124.0, 36.0, 1.21; MS calcd for $\mathrm{C}_{16} \mathrm{H}_{20} \mathrm{~N}_{3} \mathrm{Si}$ $[\mathrm{M}+\mathrm{H}]^{+}, 282.14$; found, 282.18

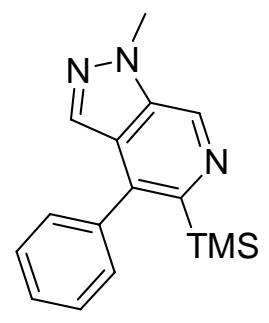




\section{1,5-dimethyl-4-phenyl-(1H)-pyrazolo[3,4-c]pyridine (10b).}

Purified by column chromatography (1:2 ethyl acetate:hexanes) to yield a white solid (0.0204 g, 11\%) ${ }^{1} \mathbf{H}$ NMR (600 MHz, $\left.\mathrm{CDCl}_{3}\right) \delta 9.13$ (s, 1H), 7.70, (s, 1H), 7.48-7.46 (m, 3H), 7.40-7.38 (m, 2H), 4.19 (s, 3H), 0.07 (s, 9H); ${ }^{13}$ C NMR (600 MHz, $\left.\mathrm{CDCl}_{3}\right) \delta$ 151.4, 138.4, 136.9 135.5, 132.6, 132.2, 129.7, 128.2, 128.0, 127.7, 36.0, 0.21; MS calcd for $\mathrm{C}_{16} \mathrm{H}_{20} \mathrm{~N}_{3} \mathrm{Si}[\mathrm{M}+\mathrm{H}]^{+}$, 282.14; found, 282.18

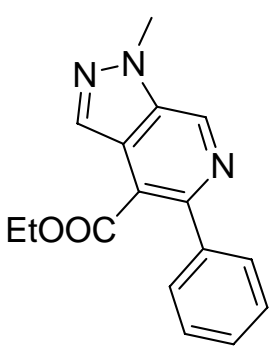

ethyl 1-methyl-5-phenyl-(1H)-pyrazolo[3,4-c]pyridine-4-carboxylate (11a).

Purified by column chromatography (1:1 ethyl acetate:hexanes) to yield a white solid (0.1247 g, 65\%) ${ }^{1}$ H NMR (600 MHz, $\left.\mathrm{CDCl}_{3}\right) \delta 9.07$ (s, 1H), 8.30 (s, 1H), 7.56-7.54 (m, 2H), 7.45-7.42 (m, 3H), 4.26 (quartet, 2H), 4.24 (s, 3H), 1.11 (t, 3H); ${ }^{13}$ C NMR (600 $\left.\mathrm{MHz}, \mathrm{CDCl}_{3}\right) \delta 167.0,149.9,140.4,135.8,134.7,132.9,129.0,128.0,127.7,117.4$, 61.4, 36.3, 13.7; MS calcd for $\mathrm{C}_{16} \mathrm{H}_{16} \mathrm{~N}_{3} \mathrm{O}_{2}[\mathrm{M}+\mathrm{H}]^{+}$, 282.12; found, 282.16

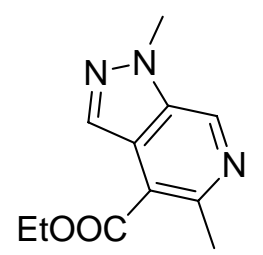

ethyl 1,5-dimethyl-(1H)-pyrazolo[3,4-c]pyridine-4-carboxylate (12).

Purified by column chromatography (3:2 ethyl acetate:hexanes) to yield a colorless residue (0.052 g, 35\%) ${ }^{1} \mathbf{H}$ NMR (600 MHz, $\mathrm{CDCl}_{3}$ ) $\delta 8.96$ (s, 1H), 8.29 (s, 1H), 4.50, (quartet, 2H), 4.20 (s, 3H), 2.95 (s, 3H), 1.50 (t, 3H); $\left.{ }^{13} \mathbf{C ~ N M R ~ ( 6 0 0 ~ M H z , ~ C D C l ~}\right) \delta$ 166.8, 151.0, 136.0, 135.7, 133.6, 128.1, 116.0, 61.5, 36.4, 24.0, 14.6; MS calcd for $\mathrm{C}_{11} \mathrm{H}_{14} \mathrm{~N}_{3} \mathrm{O}_{2}[\mathrm{M}+\mathrm{H}]^{+}$, 220.11; found, 220.34 


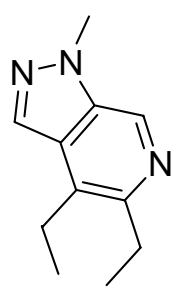

\section{4,5-diethyl-1-methyl-(1H)-pyrazolo[3,4-c]pyridine (13).}

Purified by column chromatography (1:2 ethyl acetate:hexanes) to yield a colorless oil (0.066 g, 50\%) ${ }^{1} \mathbf{H}$ NMR (600 MHz, $\left.\mathrm{CDCl}_{3}\right) \delta 8.76$ (s, 1H), 7.98 (s, 1H), 4.15 (s, 3H), 2.95 (m, 4H), 1.31 (m, 6H); ${ }^{13}$ C NMR (600 MHz, $\left.\mathrm{CDCl}_{3}\right) \delta$ 149.2, 135.7, 130.8, 130.5, 129.0, 127.2, 36.0, 26.9, 22.7, 15.3, 13.9; MS calcd for $\mathrm{C}_{11} \mathrm{H}_{16} \mathrm{~N}_{3}[\mathrm{M}+\mathrm{H}]^{+}$, 190.13; found, 190.34

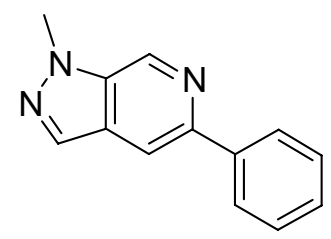

\section{1-methyl-5-phenyl-(1H)-pyrazolo[3,4-c]pyridine (14).}

Purified by column chromatography (1:1 ethyl acetate:hexanes) to yield a clear colorless residue (0.066 g, 46\%) ${ }^{1} \mathbf{H}$ NMR (600 MHz, $\mathrm{CDCl}_{3}$ ) $\delta 9.04$ (s, 1H), 8.05, (s, 1H), 8.00 (m, 3H), 7.47 (m, 2H), 7.38 (m, 1H), 4.19 (s, 3H); ${ }^{13}$ C NMR (600 MHz, $\left.\mathrm{CDCl}_{3}\right) \delta$ 147.8, 139.9 138.9, 136.0, 133.1, 132.6, 128.7, 128.0, 126.8, 110.9, 36.1; MS calcd for $\mathrm{C}_{13} \mathrm{H}_{12} \mathrm{~N}_{3}[\mathrm{M}+\mathrm{H}]^{+}$, 210.10; found, 210.14

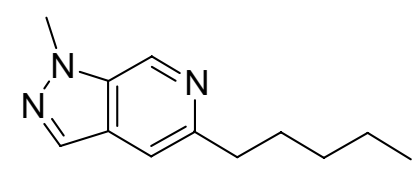

\section{1-methyl-5-pentyl-(1H)-pyrazolo[3,4-c]pyridine (15).}

Purified by column chromatography (1:1 ethyl acetate:hexanes) to yield a colorless oil (0.0593 g, 43\%) ${ }^{1} \mathbf{H}$ NMR (600 MHz, $\mathrm{CDCl}_{3}$ ) $\delta 8.89$ (s, 1H), 7.93 (s, 1H), 7.41 (s, 1H), 4.16 (s, 3H), 2.90 (t, 2H), 1.77 (quint, 2H), 1.38-1.34 (m, 4 H), 0.90 (t, 3H); ${ }^{13}$ C NMR $\left(600 \mathrm{MHz}, \mathrm{CDCl}_{3}\right) \delta 151.5,135.5,132.7,131.6,128.7,112.1,37.8,36.0,31.5,30.1$, 22.5, 14.0; MS calcd for $\mathrm{C}_{12} \mathrm{H}_{18} \mathrm{~N}_{3}[\mathrm{M}+\mathrm{H}]^{+}$, 204.15; found, 204.20 


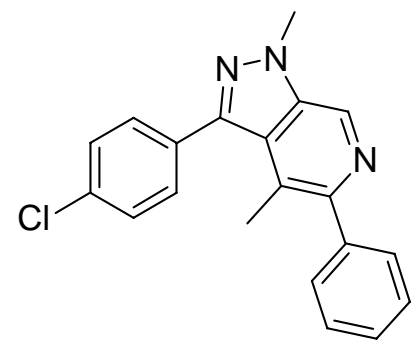

3-(4-chlorophenyl)-1,4-dimethyl-5-phenyl-(1H)-pyrazolo[3,4-c]pyridine (16a).

Purified by column chromatography (1:10, acetone:DCM) to yield a tan solid (0.0481 g, 55\%). ${ }^{1}$ H NMR (600 MHz, $\left.\mathrm{CDCl}_{3}\right) \delta 8.90$ (s, 1H), 7.57-7.51 (m, 4H), 7.47-7.7.43 (m, 4H), 7.38 (m, 1H,), 4.24 (s, 3H), 2.33 (s, 3H); $\left.{ }^{13} \mathrm{C} \mathrm{NMR} \mathrm{(600} \mathrm{MHz,} \mathrm{CDCl}_{3}\right) \delta$ 149.0, 144.5, 140.6, 136.9, 134.5, 132.2, 131.3, 130.9, 129.8, 128.4, 128.1, 127.4, 126.7, 122.7, 36.1, 17.6; MS calcd for $\mathrm{C}_{20} \mathrm{H}_{17} \mathrm{ClN}_{3}[\mathrm{M}+\mathrm{H}]^{+}$, 334.11; found, 334.17

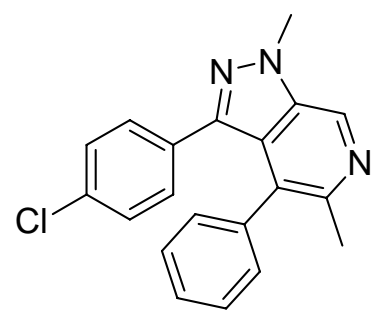

\section{3-(4-chlorophenyl)-1,5-dimethyl-4-phenyl-(1H)-pyrazolo[3,4-c]pyridine (16b).}

Purified by column chromatography (1:4, acetone:DCM) to yield a tan solid (0.0518 g, 29\%). ${ }^{1} \mathbf{H}$ NMR (400 MHz, $\left.\mathrm{CDCl}_{3}\right) \delta 8.91$ (s, 1H), $7.26(\mathrm{~m}, 1 \mathrm{H}), 7.18$ (m, 2H), 7.04 (d, 2H, $J=6.8 \mathrm{~Hz}$ ), 6.96 (d, 2H, $J=8.4 \mathrm{~Hz}$ ), 6.90 (d, 2H, $J=8.4 \mathrm{~Hz}), 4.24$ (s, 3H), 2.53 (s, 3H); ${ }^{13} \mathrm{C}$ NMR (600 MHz, $\left.\mathrm{CDCl}_{3}\right) \delta$ 145.3, 143.6, 136.9, 136.7, 133.1, 131.9, 131.0, 130.1, 129.8, 128.4, 128.0, 127.6, 127.5, 125.4, 36.1, 22.0; MS calcd for $\mathrm{C}_{20} \mathrm{H}_{17} \mathrm{ClN}_{3}$ $[\mathrm{M}+\mathrm{H}]^{+}, 334.11$; found, 334.17<smiles>Cn1nc(-c2ccc(Cl)cc2)c2c(-c3ccccc3)c(-c3ccccc3)ncc21</smiles>

3-(4-chlorophenyl)-4,5-diphenyl-1-methyl-(1H)-pyrazolo[3,4-c]pyridine (17).

Purified by column chromatography (1:19 acetone:DCM) to yield a tan solid (0.0967 g, 49\%). ${ }^{1}$ H NMR (600 MHz, $\left.\mathrm{CDCl}_{3}\right) \delta 9.077$ (s, 1H), 7.29-7.26 (m, 2H), 7.20-7.17 (m, 
3H), 7.14-7.10 (m, 1H), 7.00-6.96 (m, 4H), 6.90-6.87 (m, 4H), 4.30 (s, 3H); ${ }^{13}$ C NMR (600 MHz, $\left.\mathrm{CDCl}_{3}\right) \delta 147.6,144.6,140.3,137.1,136.1,133.3,132.3,131.1,130.7,130.4$, 130.3, 128.7, 127.7, 127.6, 127.5, 127.2, 126.8, 125.4, 36.2; MS calcd for $\mathrm{C}_{25} \mathrm{H}_{19} \mathrm{ClN}_{3}$, 396.13; found, 396.12

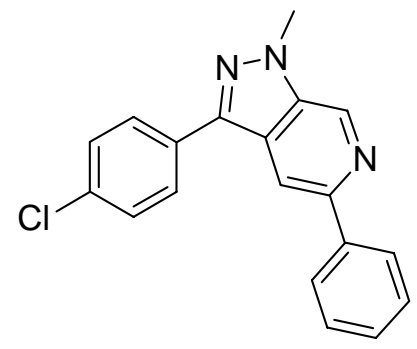

3-(4-chlorophenyl)-1-methyl-5-phenyl-(1H)-pyrazolo[3,4-c]pyridine (18).

Purified by column chromatography (1:1 ethyl acetate:hexanes) to yield an off-white solid (0.062 g, 39\%) ${ }^{1} \mathbf{H}$ NMR (600 MHz, CDCl 3 ) $\delta 9.02$ (s, 1H), 8.17 (s, 1H), 8.02 (d, $2 \mathrm{H}, J=7.8 \mathrm{~Hz}$ ), 7.92-7.90 (m, 2H), 7.51-7.49 (m, 4H) 7.40 (m, 1H), 4.23 (s, 3H); ${ }^{13} \mathrm{C}$ NMR (600 MHz, $\left.\mathrm{CDCl}_{3}\right) \delta 148.7,142.7,139.9,137.4,134.2,133.6,131.2,129.2,128.8$, 128.3, 129.1, 126.9, 126.4, 110.9, 36.2; MS calcd for $\mathrm{C}_{19} \mathrm{H}_{15} \mathrm{ClN}_{3}[\mathrm{M}+\mathrm{H}]^{+}, 320.10$; found, 320.36

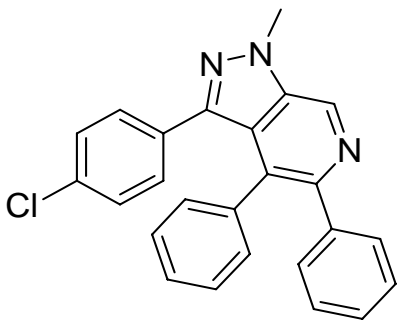

3-(4-chlorophenyl)-4,5-diphenyl-2-methyl-(2H)-pyrazolo[3,4-c]pyridine (19).

Reaction run at $0.025 \mathrm{M}$ ( $20 \mathrm{~mL}$ acetonitrile) due to the lower solubility of $\mathbf{4 b}$. Purified by column chromatography (1:19 acetone:DCM) to yield a tan solid (0.0835 g, 42\%). ${ }^{1}$ H NMR (600 MHz, $\mathrm{CDCl}_{3}$ ) $\delta 9.37$ (s, 1H), 7.33-7.31 (m, 2H), 7.18-7.14 (m, 3H), 7.07 (d, 2H, $J=8.4 \mathrm{~Hz}$ ), 7.19 (t, 1H), 6.80 (t 1H), 6.81-6.78 (m, 4H), 4.09 (s, 1H); ${ }^{13}$ C NMR $\left(600 \mathrm{MHz}, \mathrm{CDCl}_{3}\right) \delta 145.8,144.1,142.7,140.4,136.0,135.9,134.7,131.2,130.5,130.3$, 128.1, 127.6, 127.5, 127.4, 126.6, 126.2, 123.7, 39.0; MS calcd for $\mathrm{C}_{25} \mathrm{H}_{19} \mathrm{ClN}_{3}$, 396.13; found, 396.12 


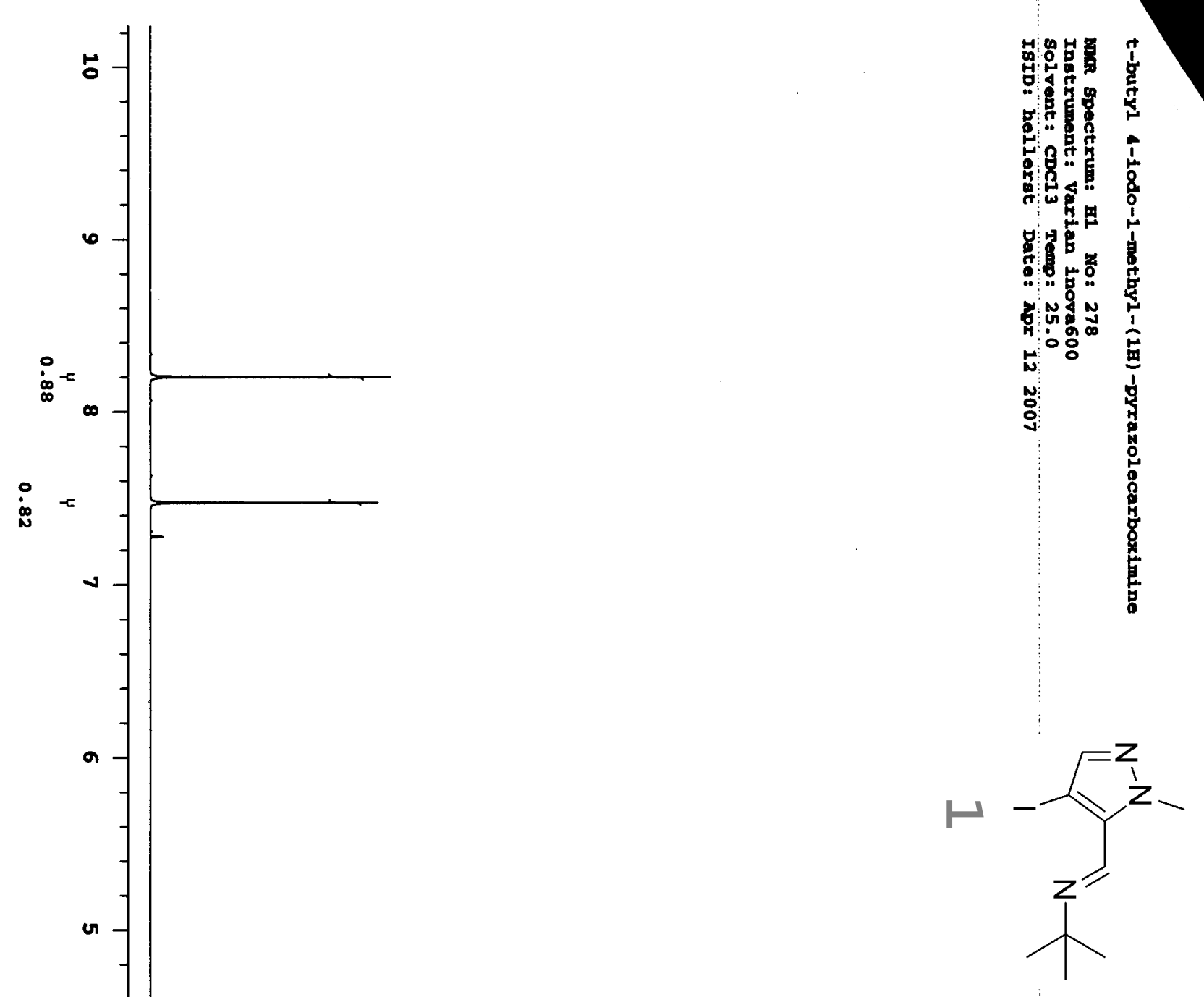




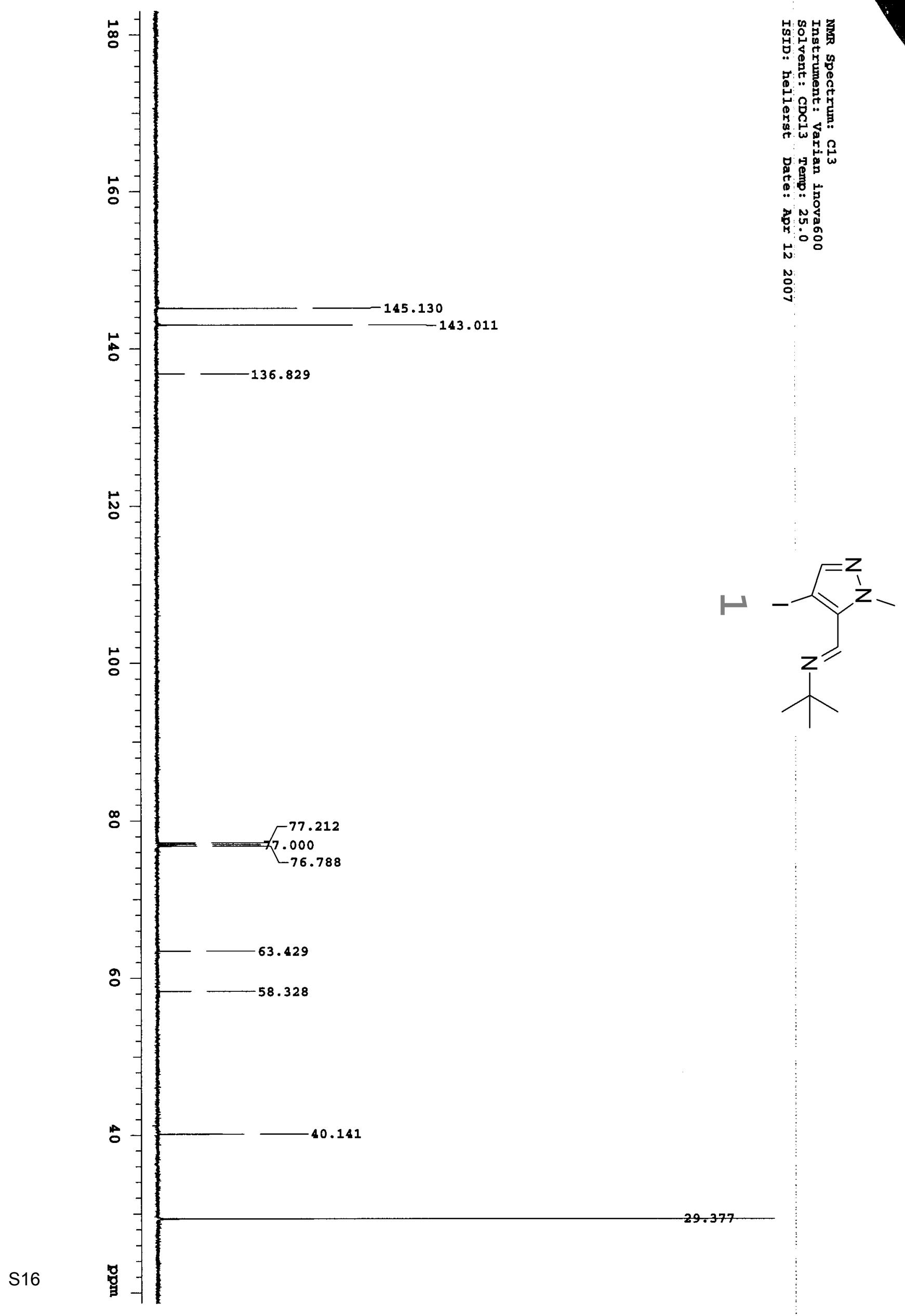




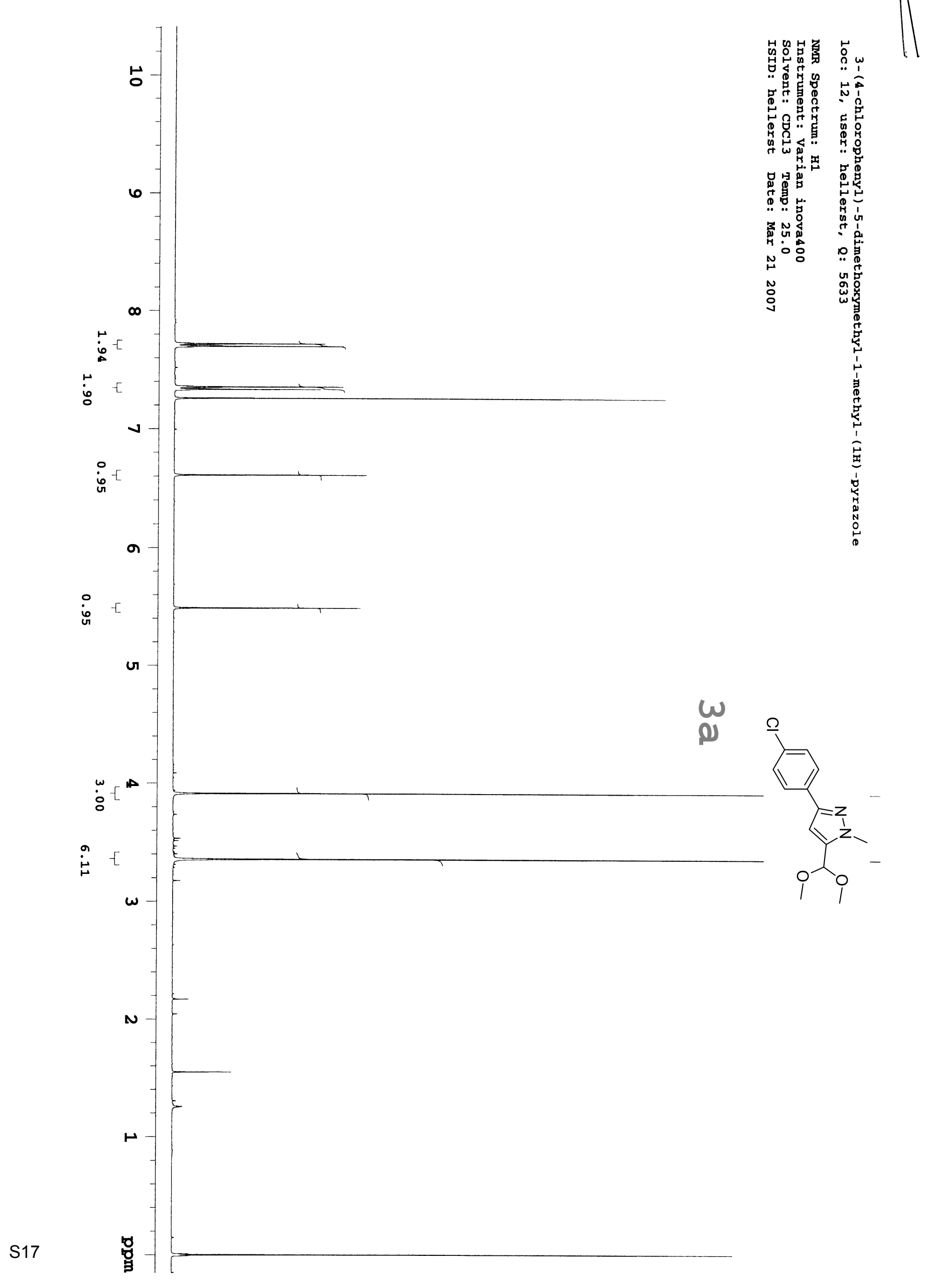




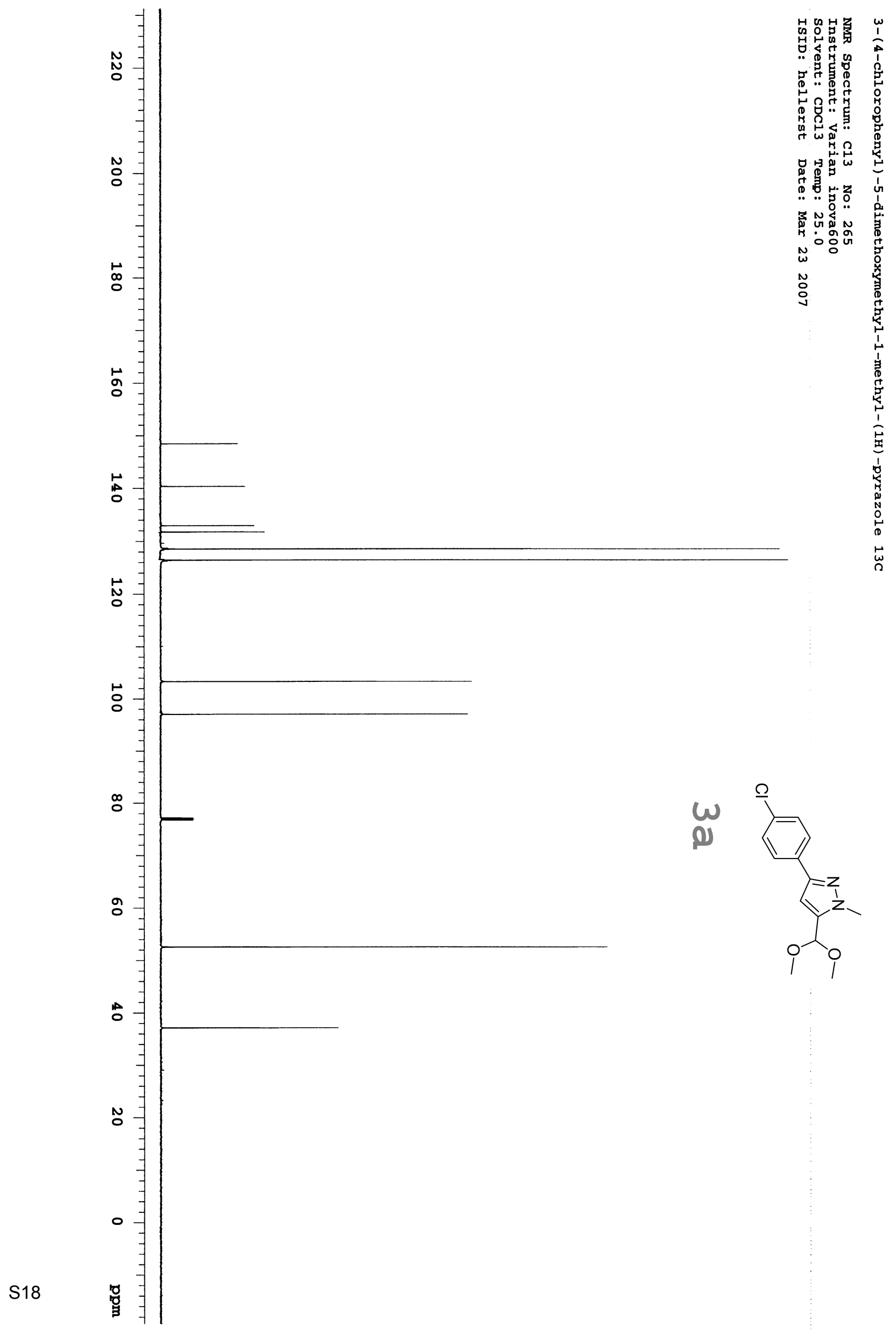



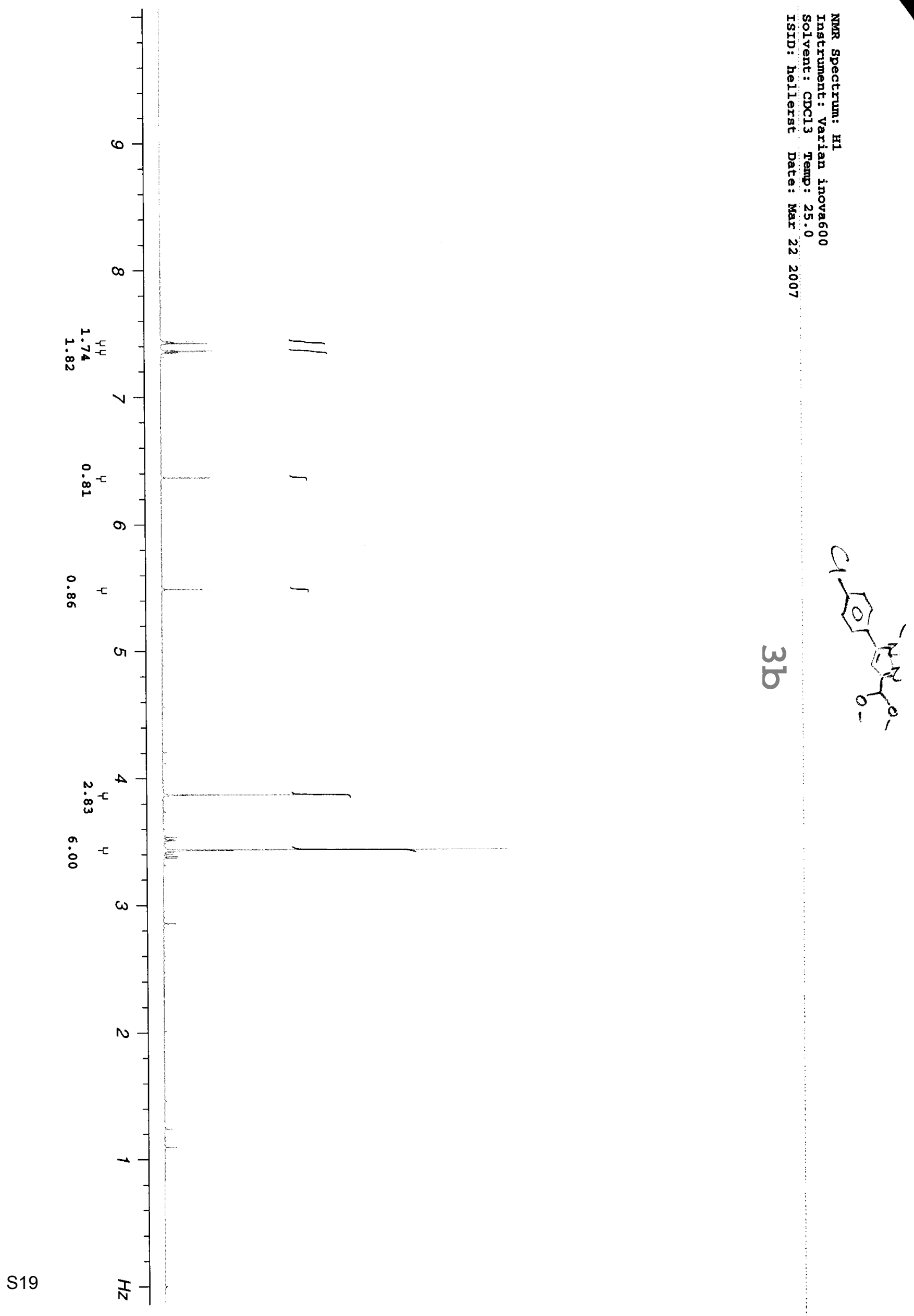

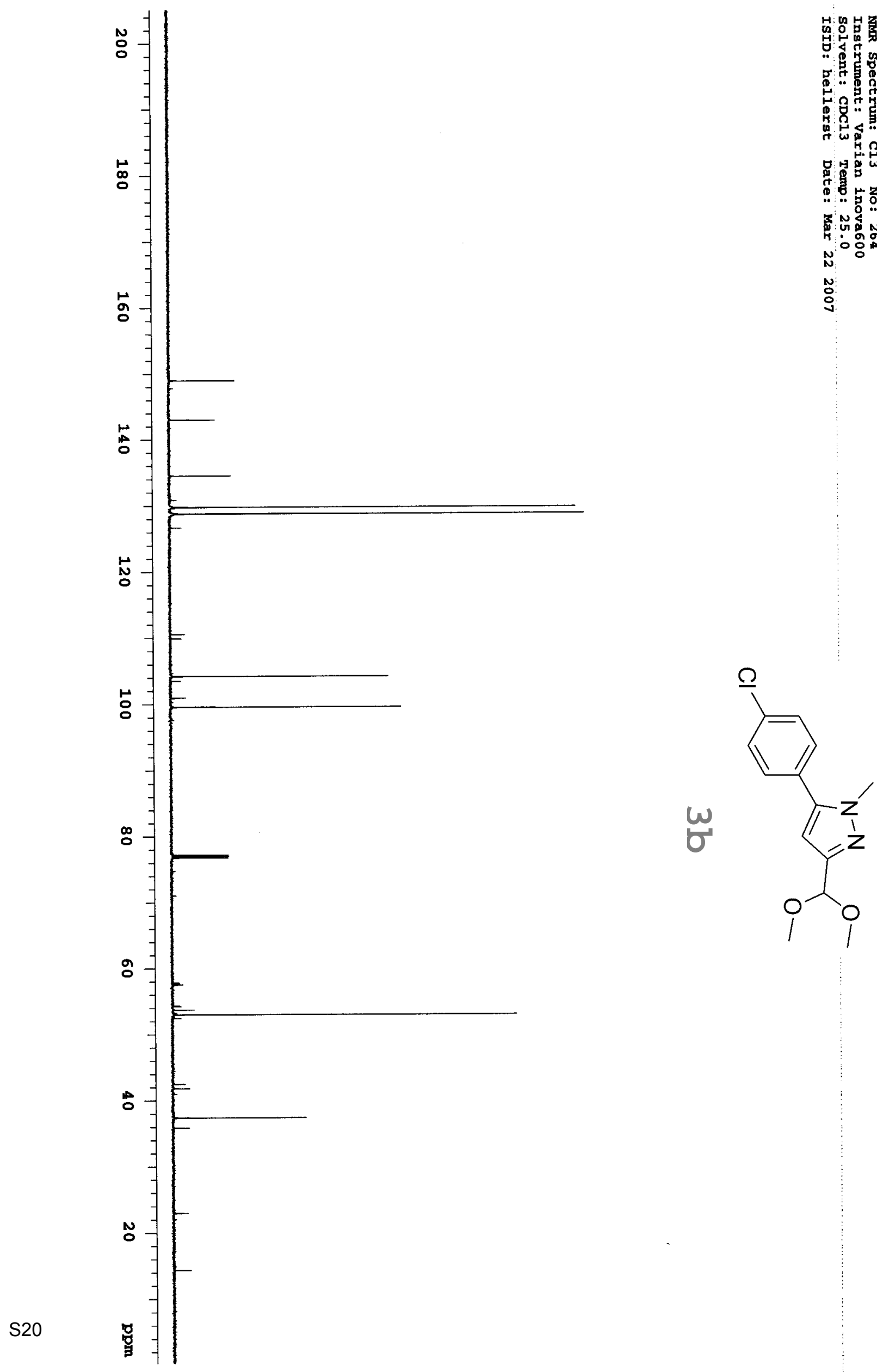


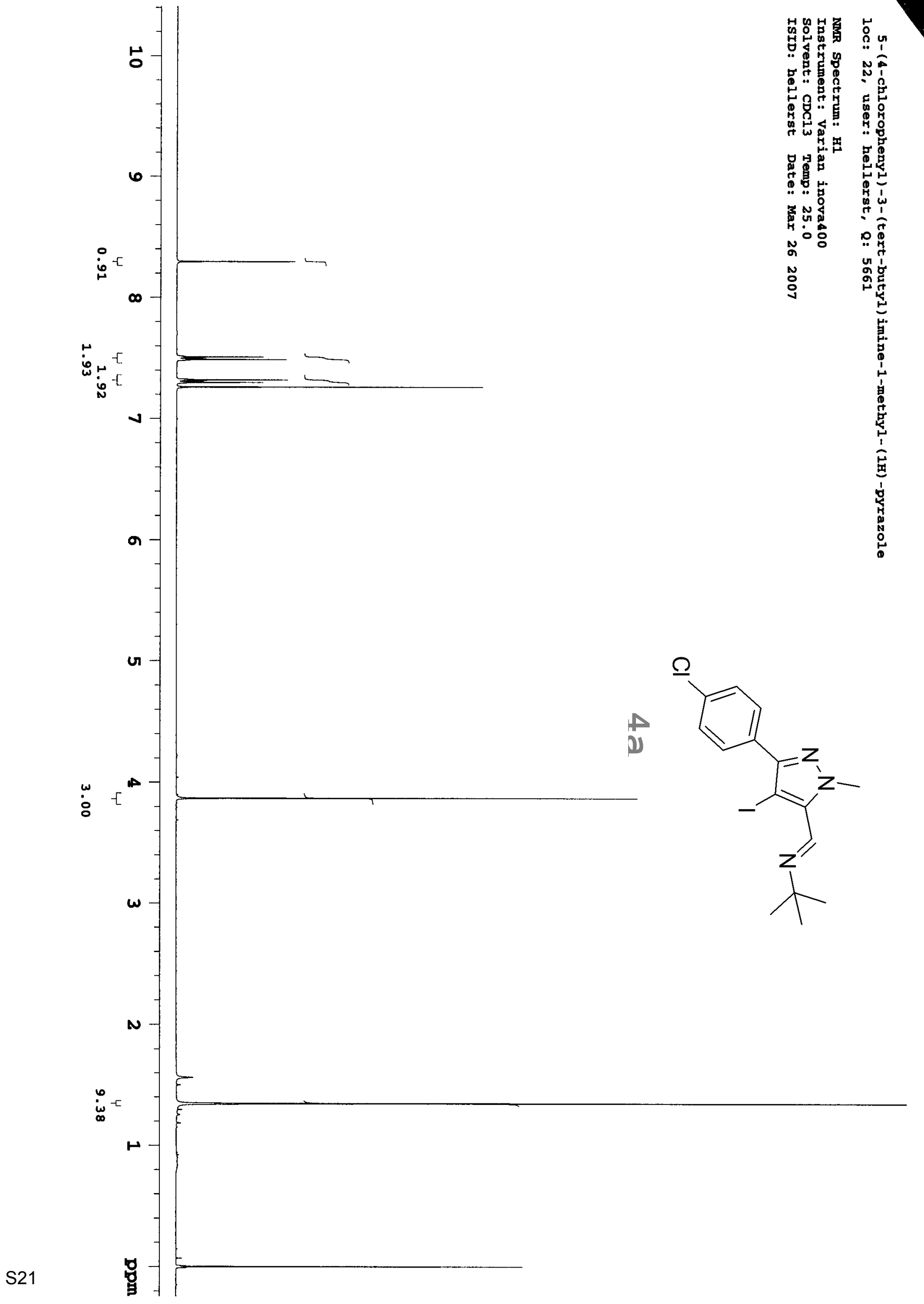




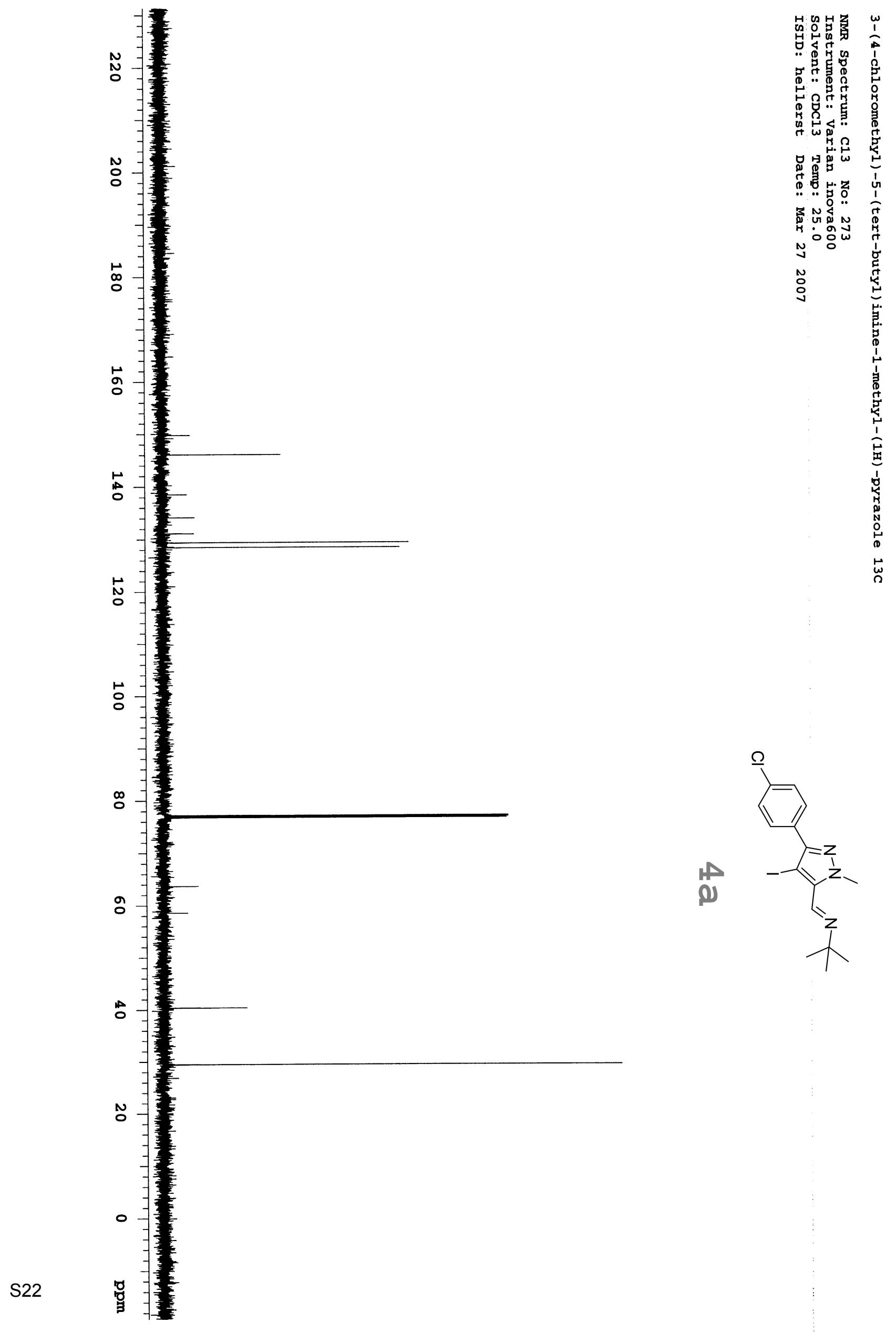




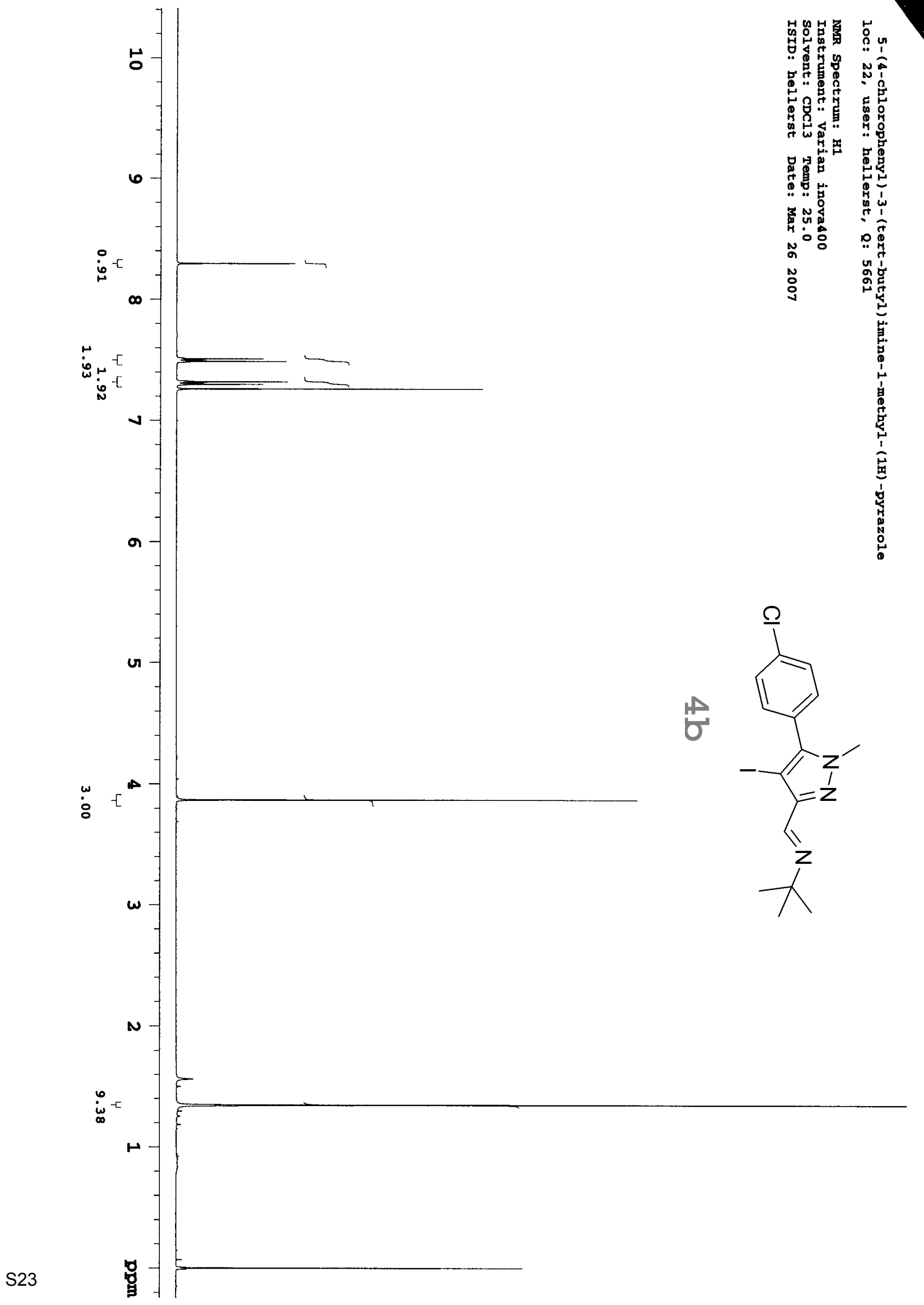




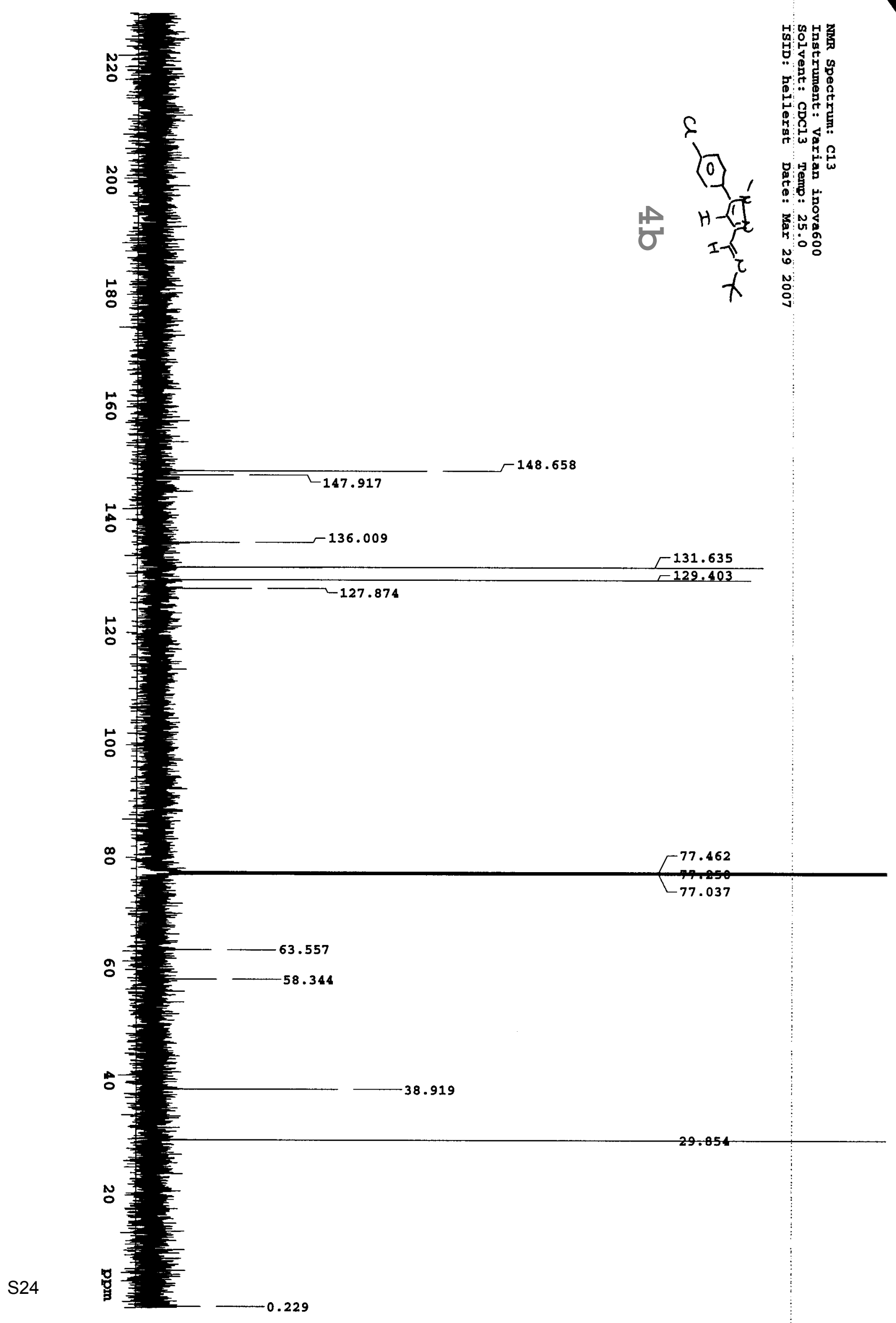



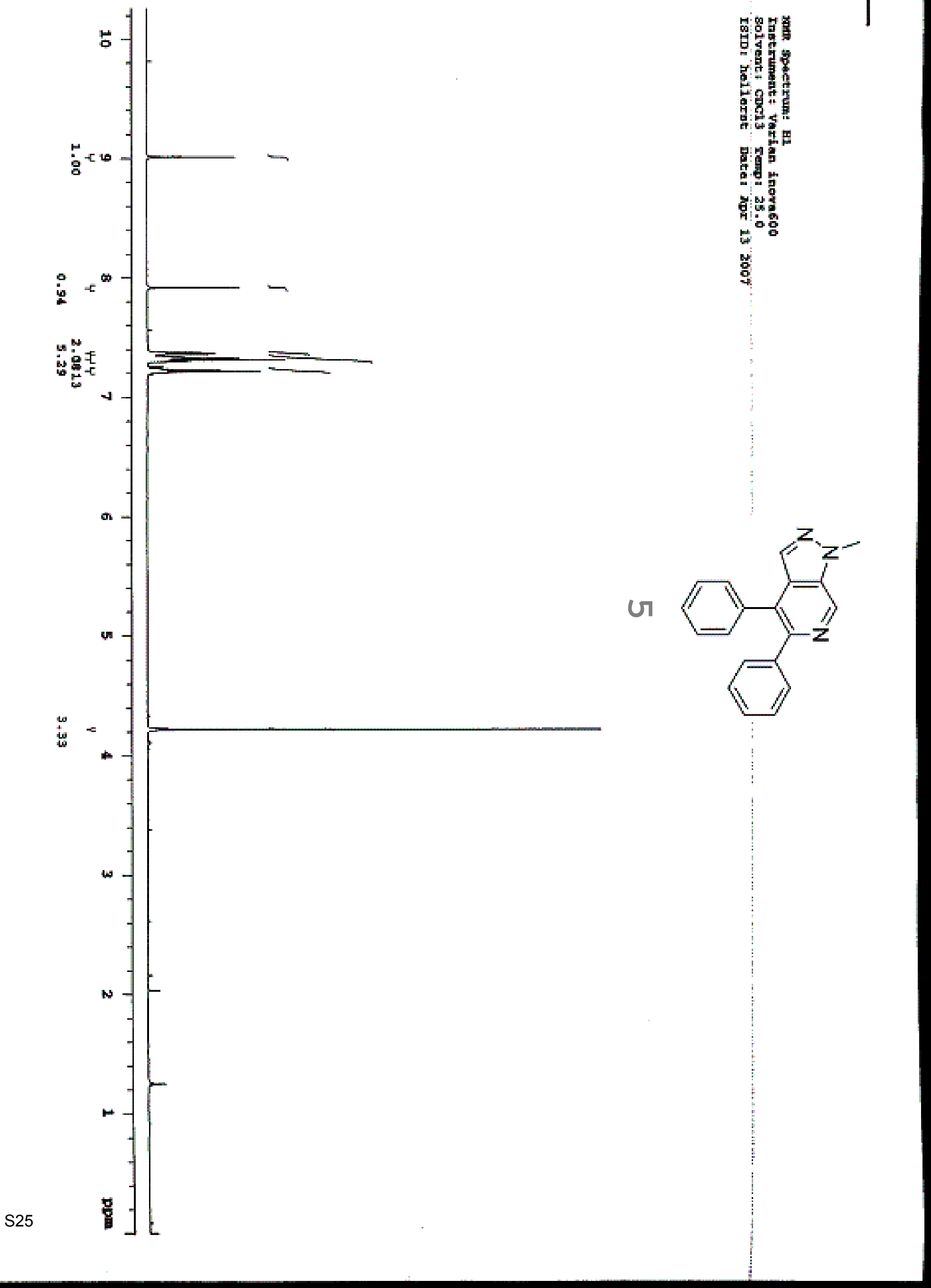


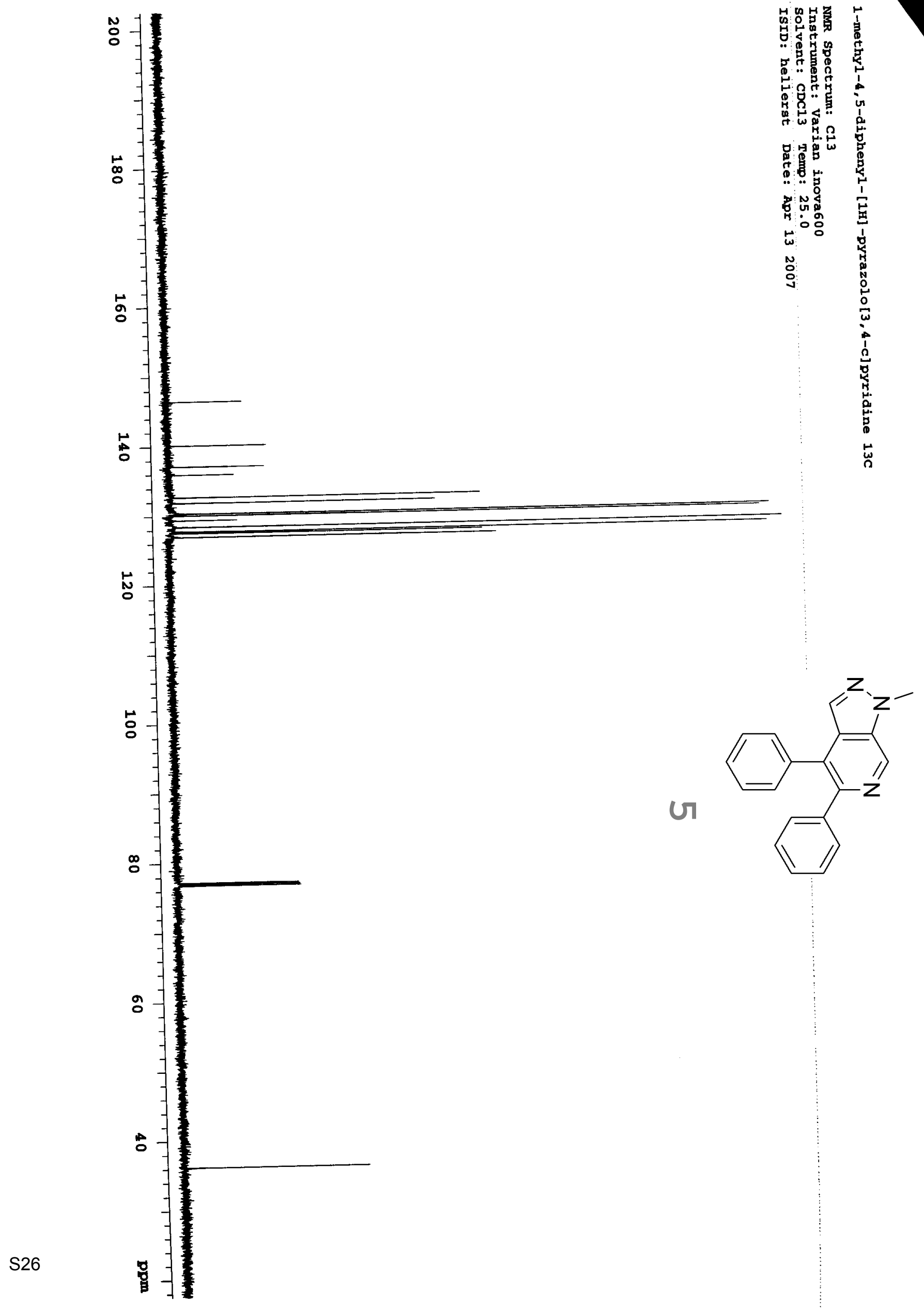




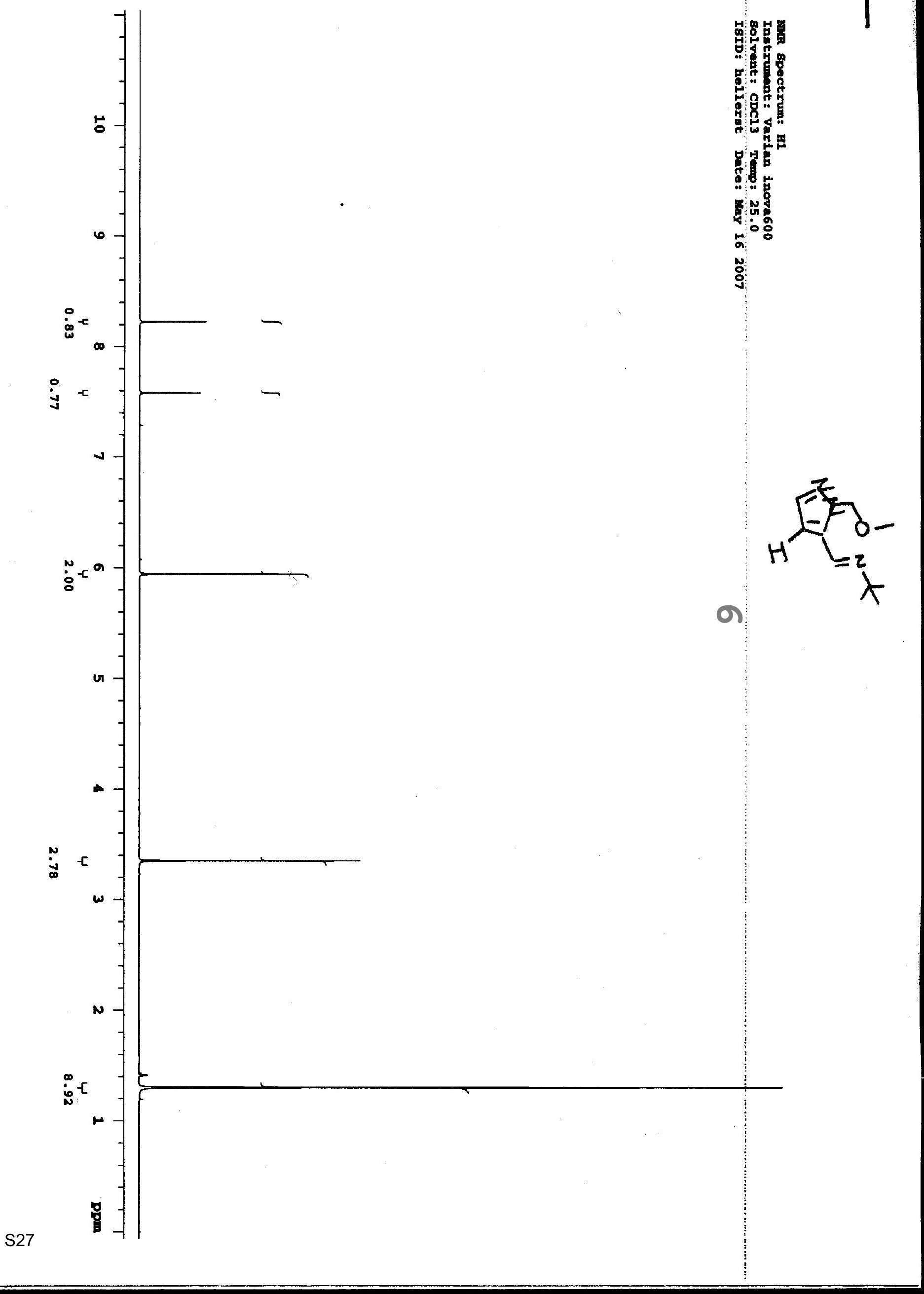




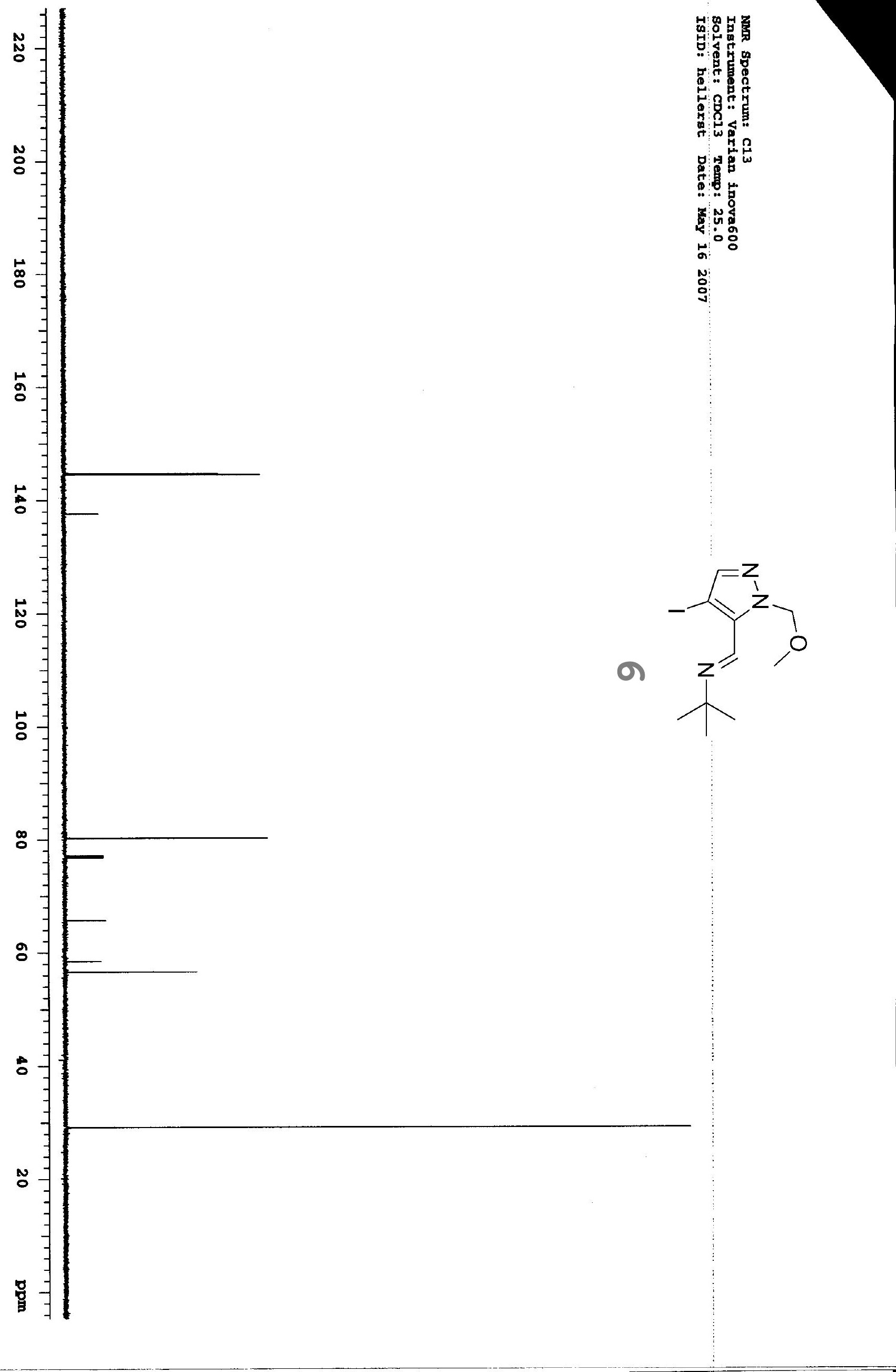





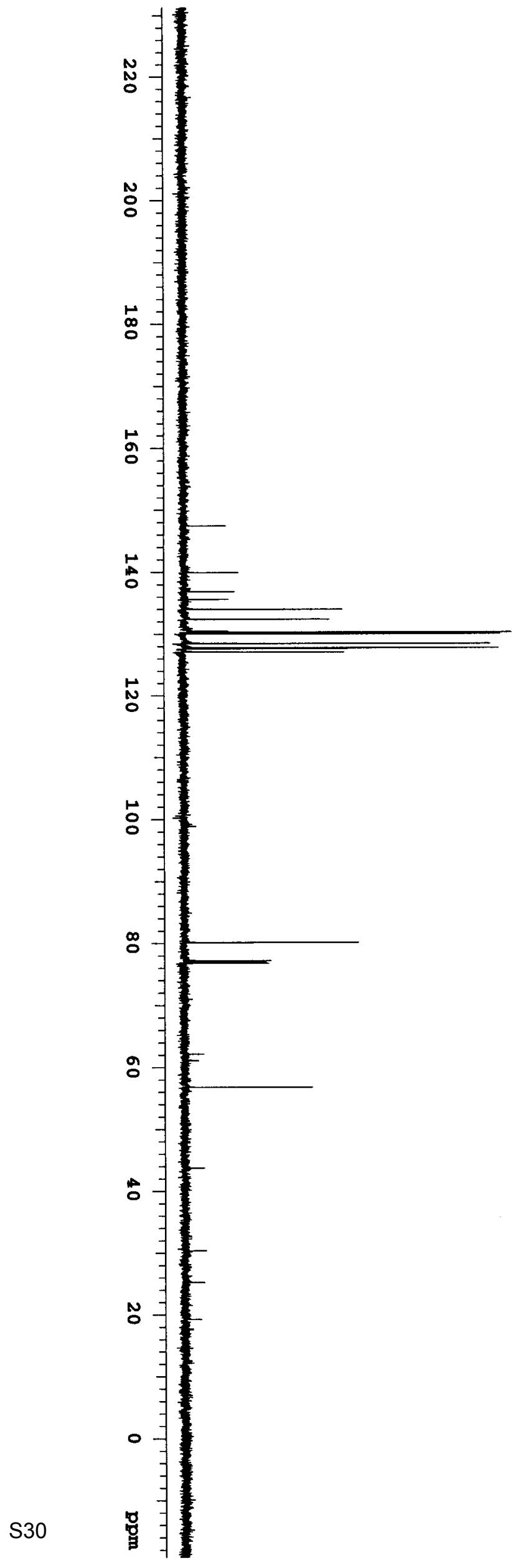

:

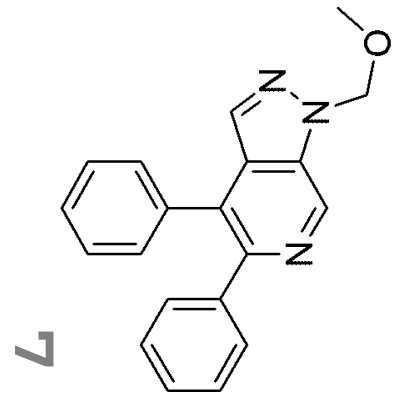




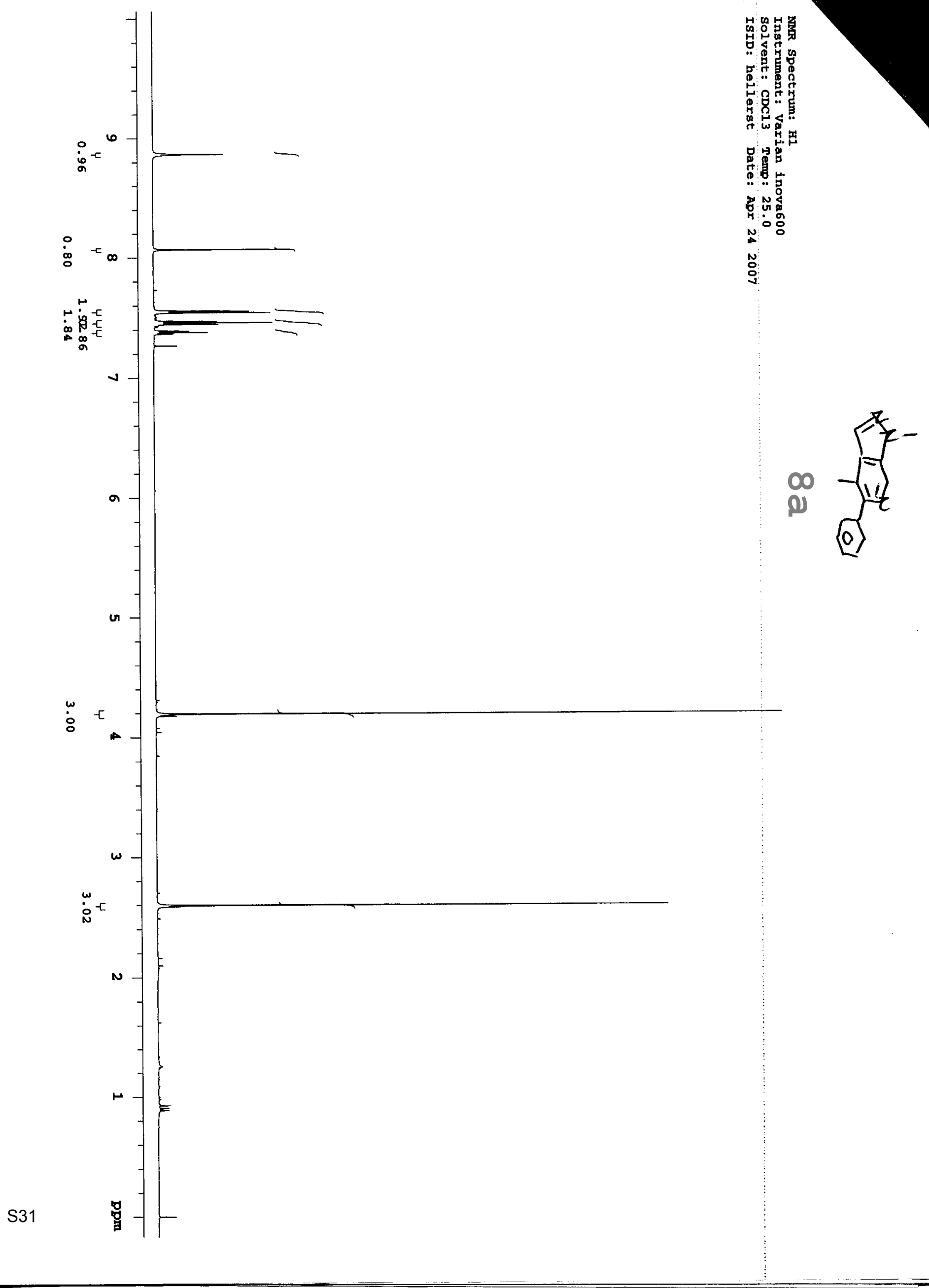




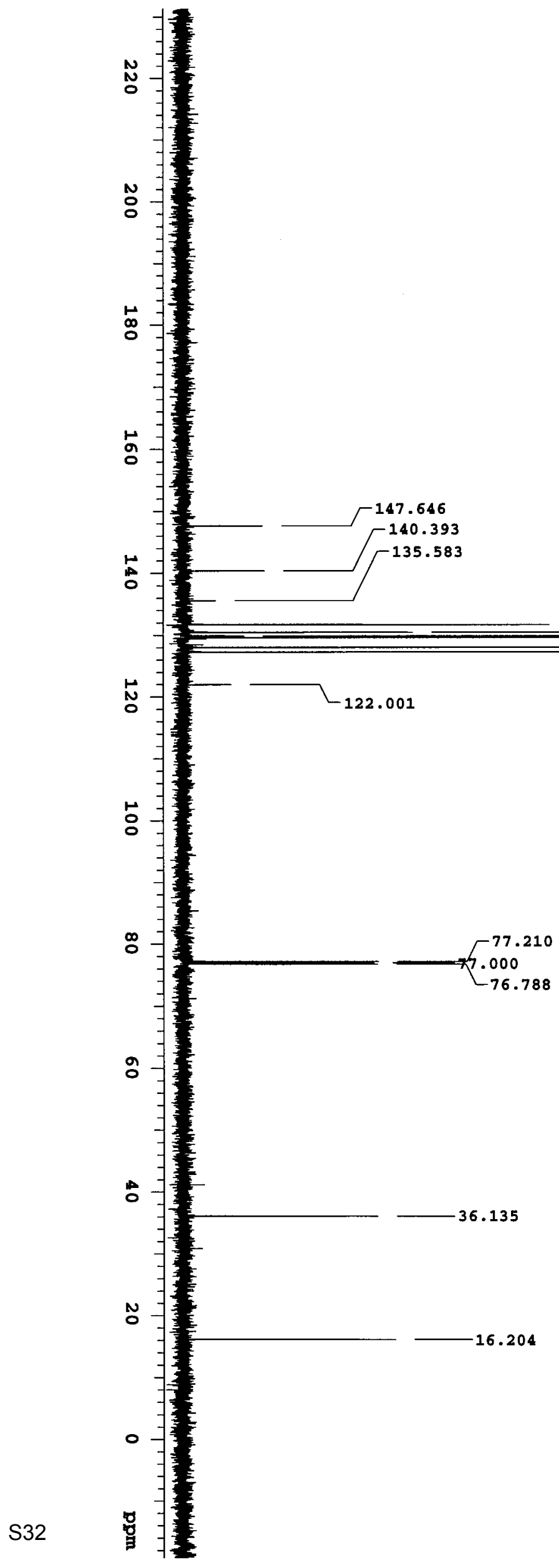

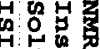

$\forall 4$

듀:

要

...

$+\omega$

궁

+.

N

品

N०\%

옹 

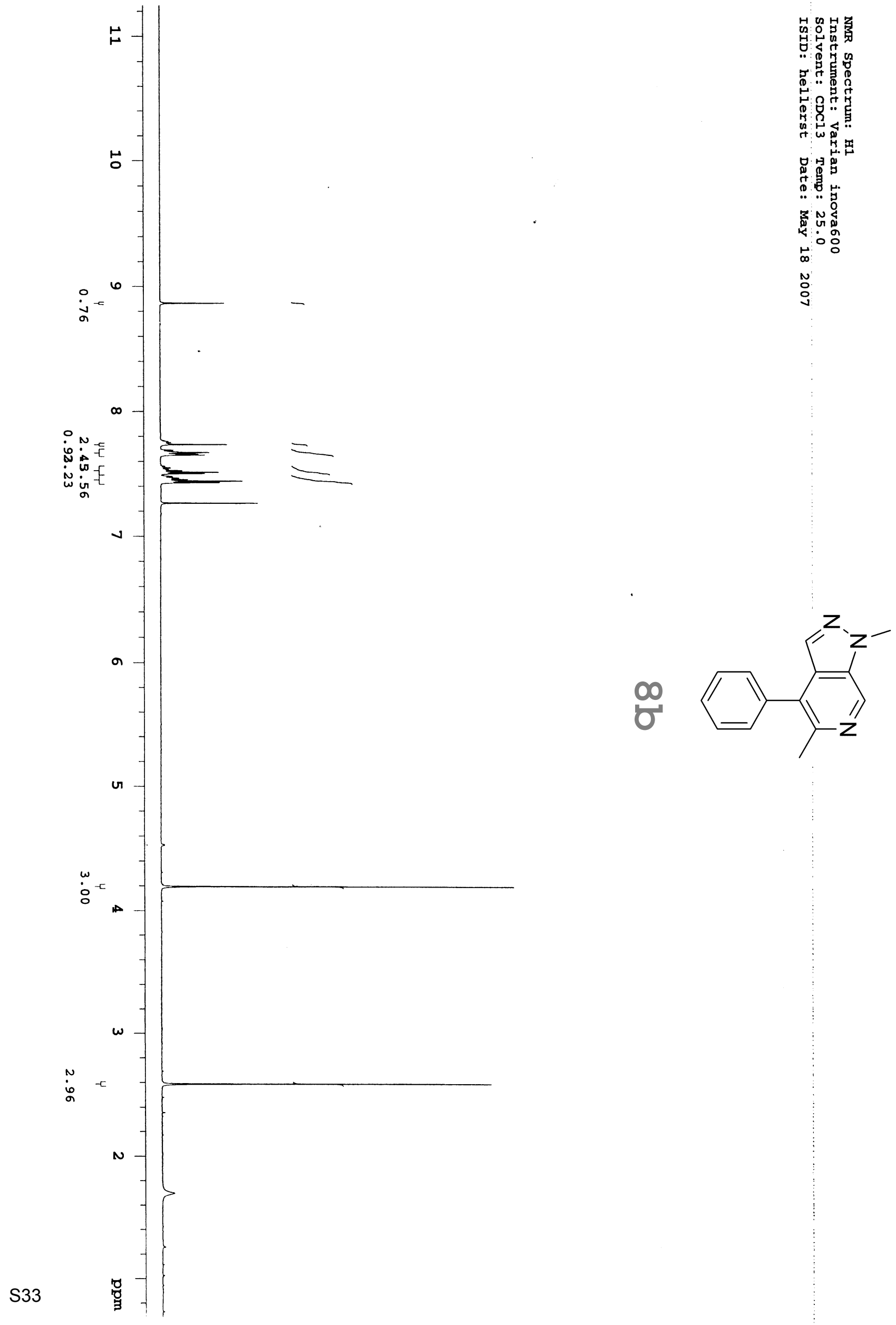

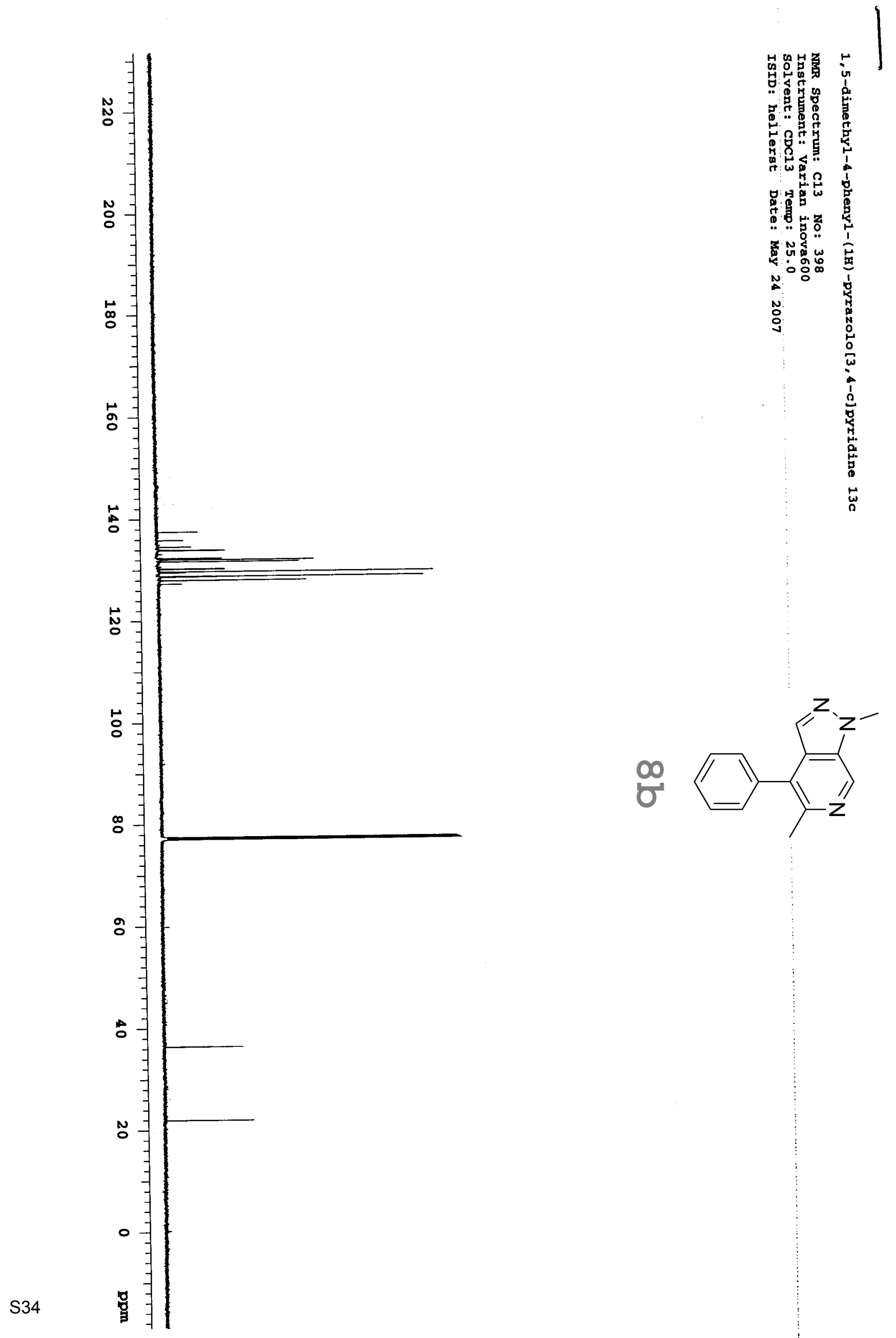


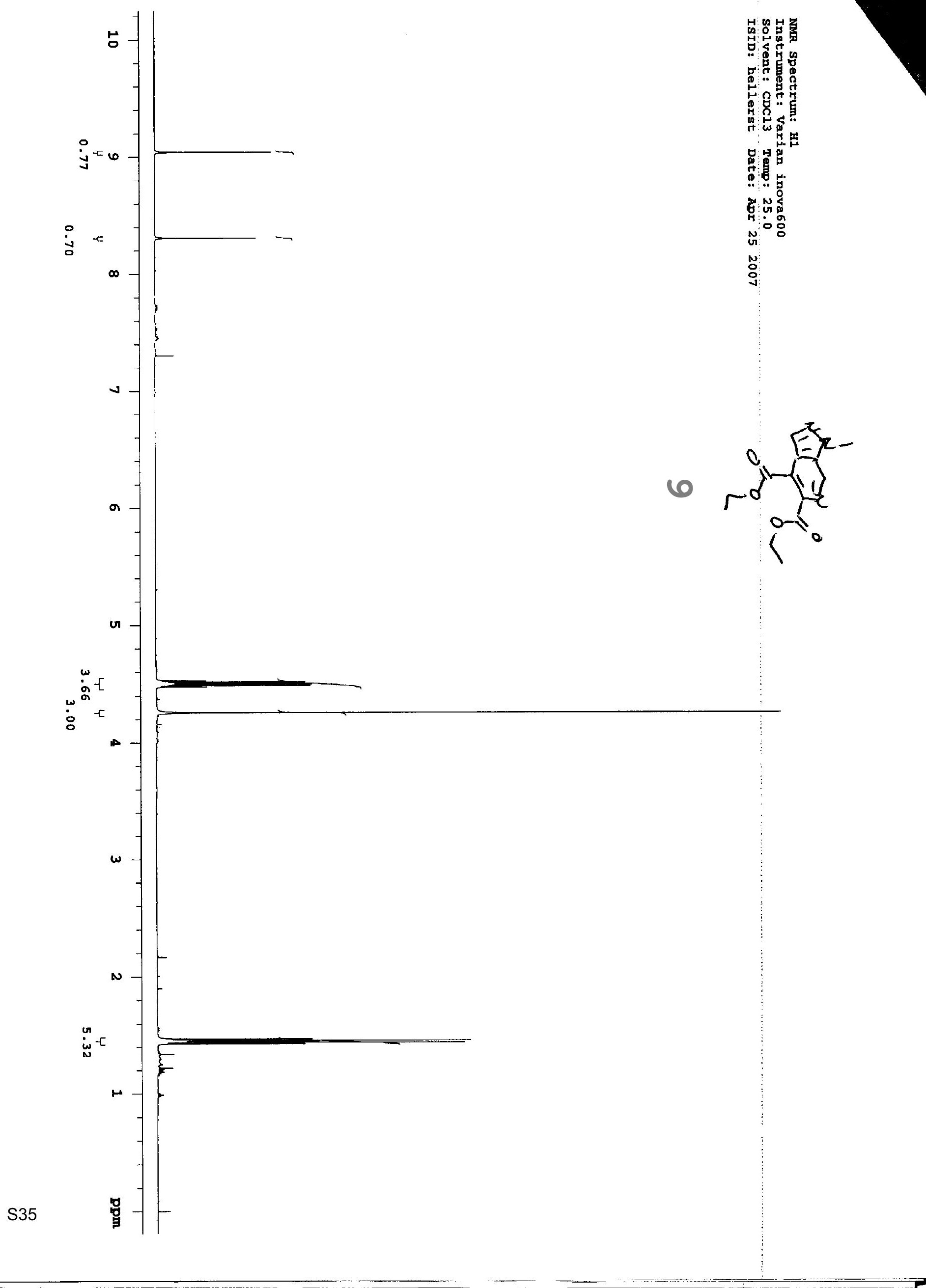



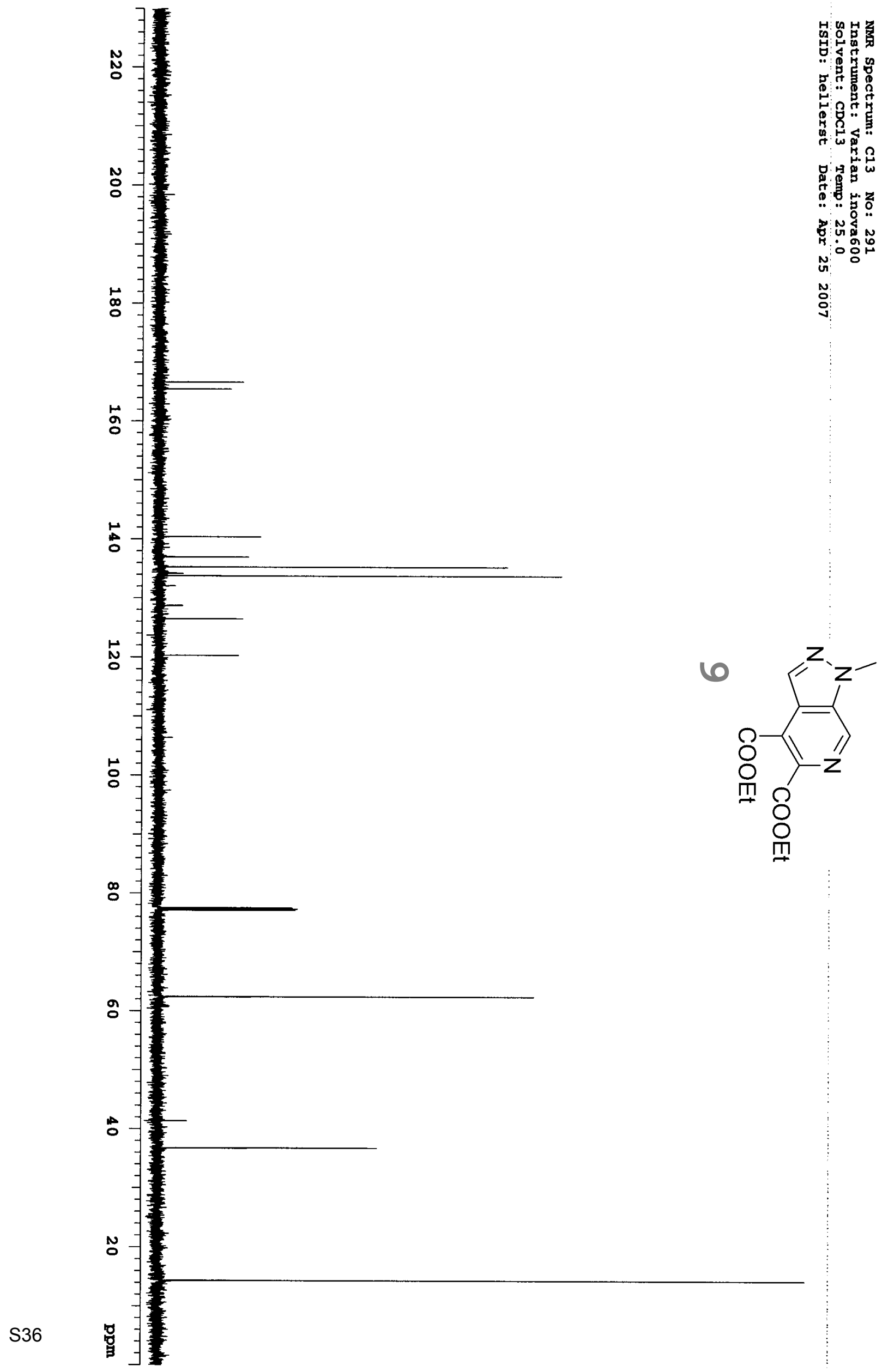

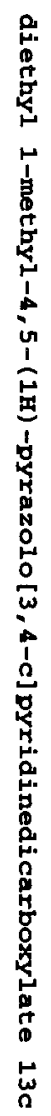

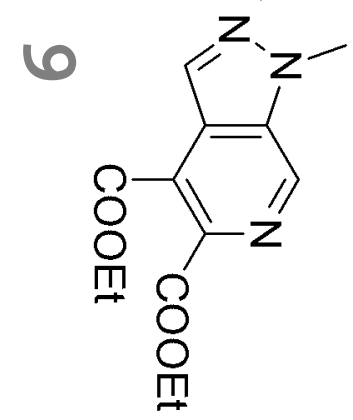




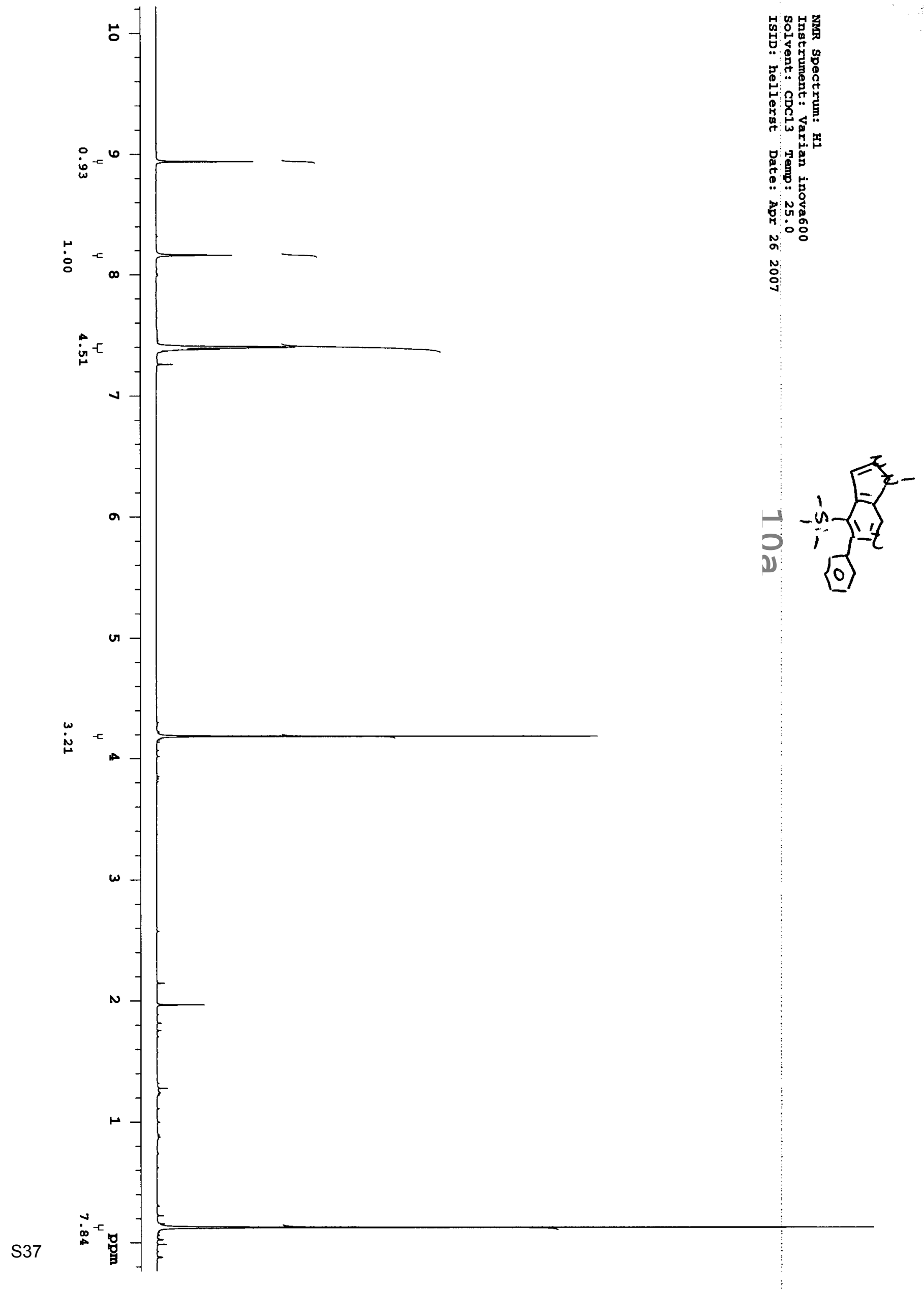




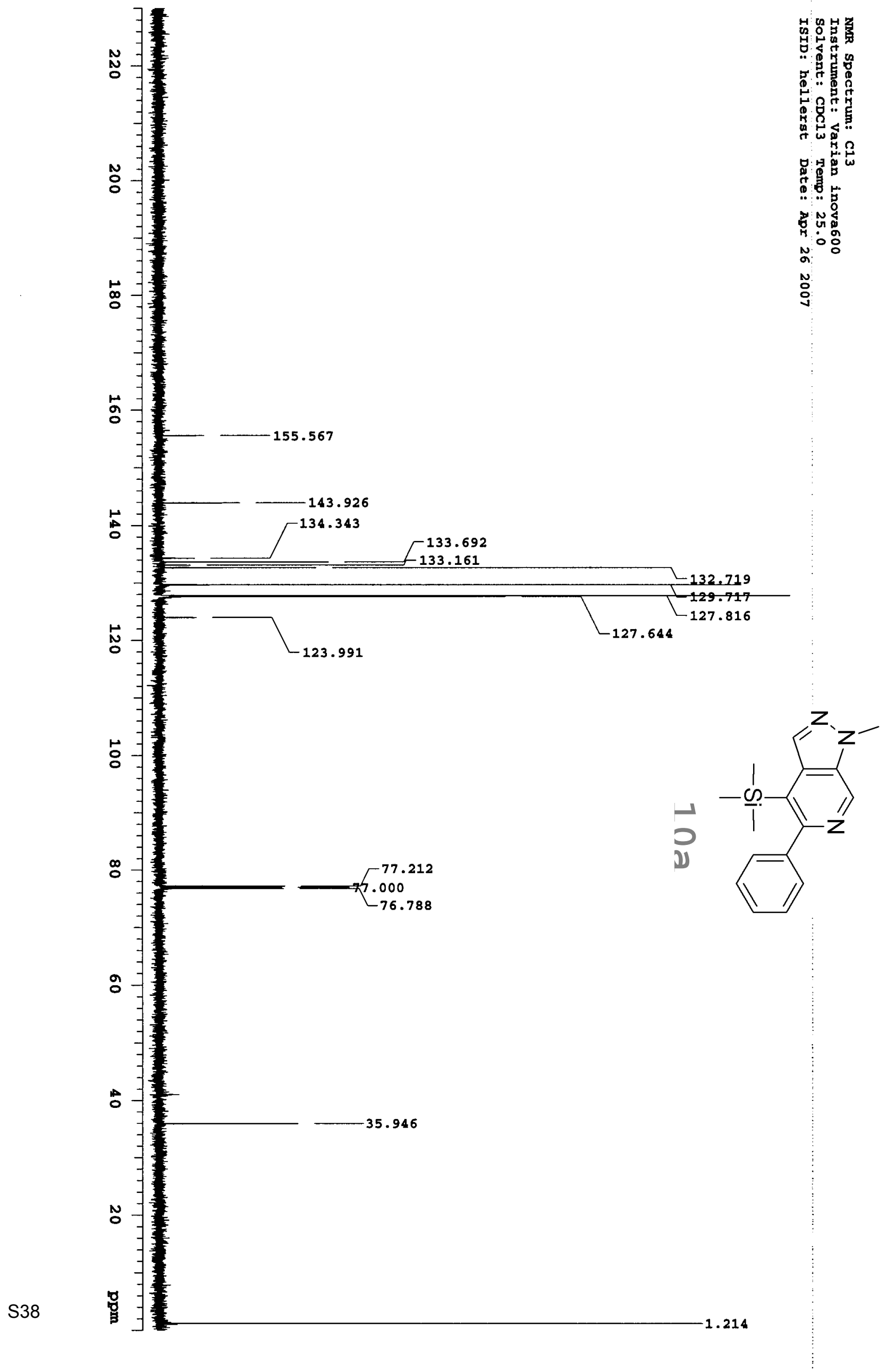




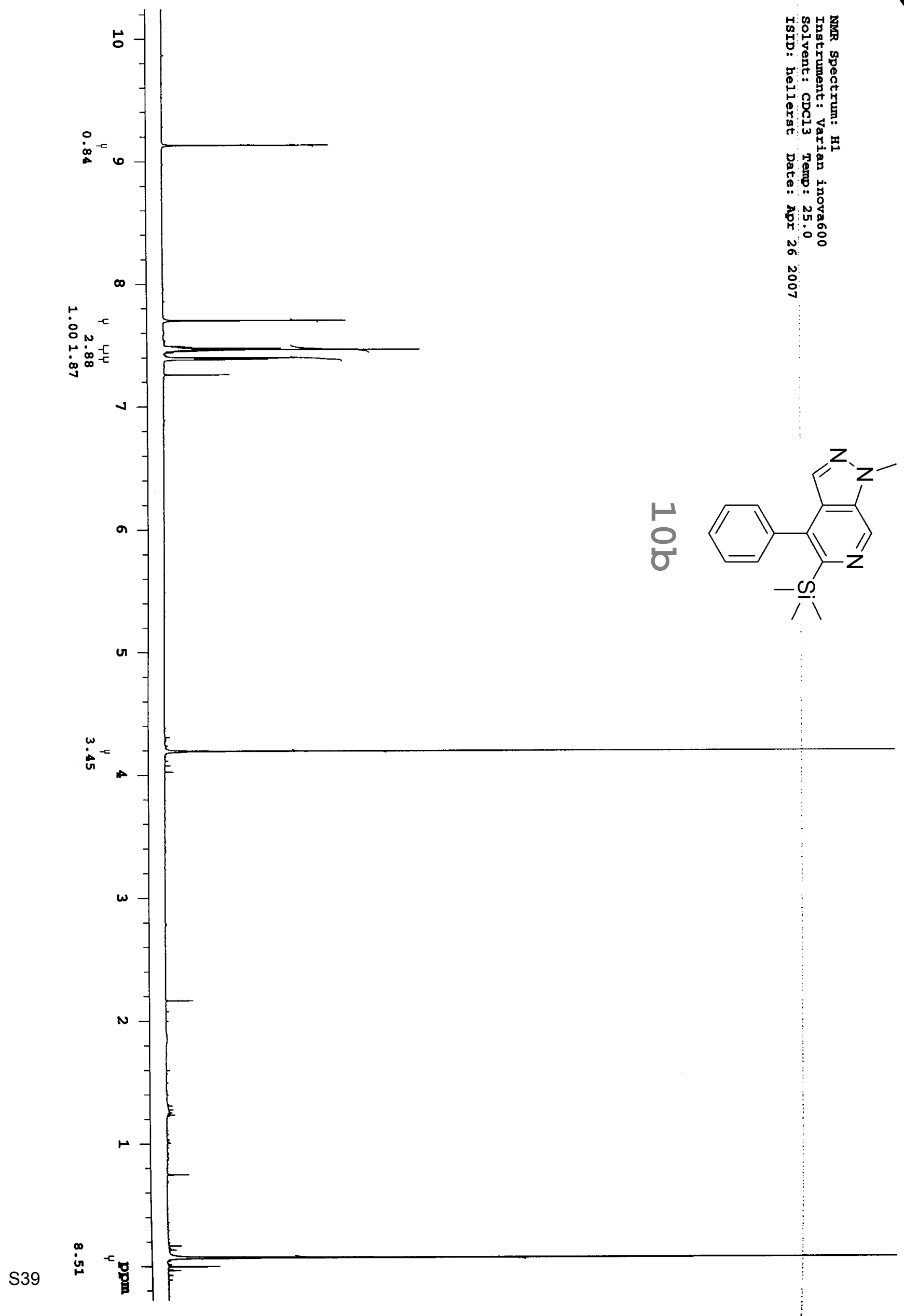




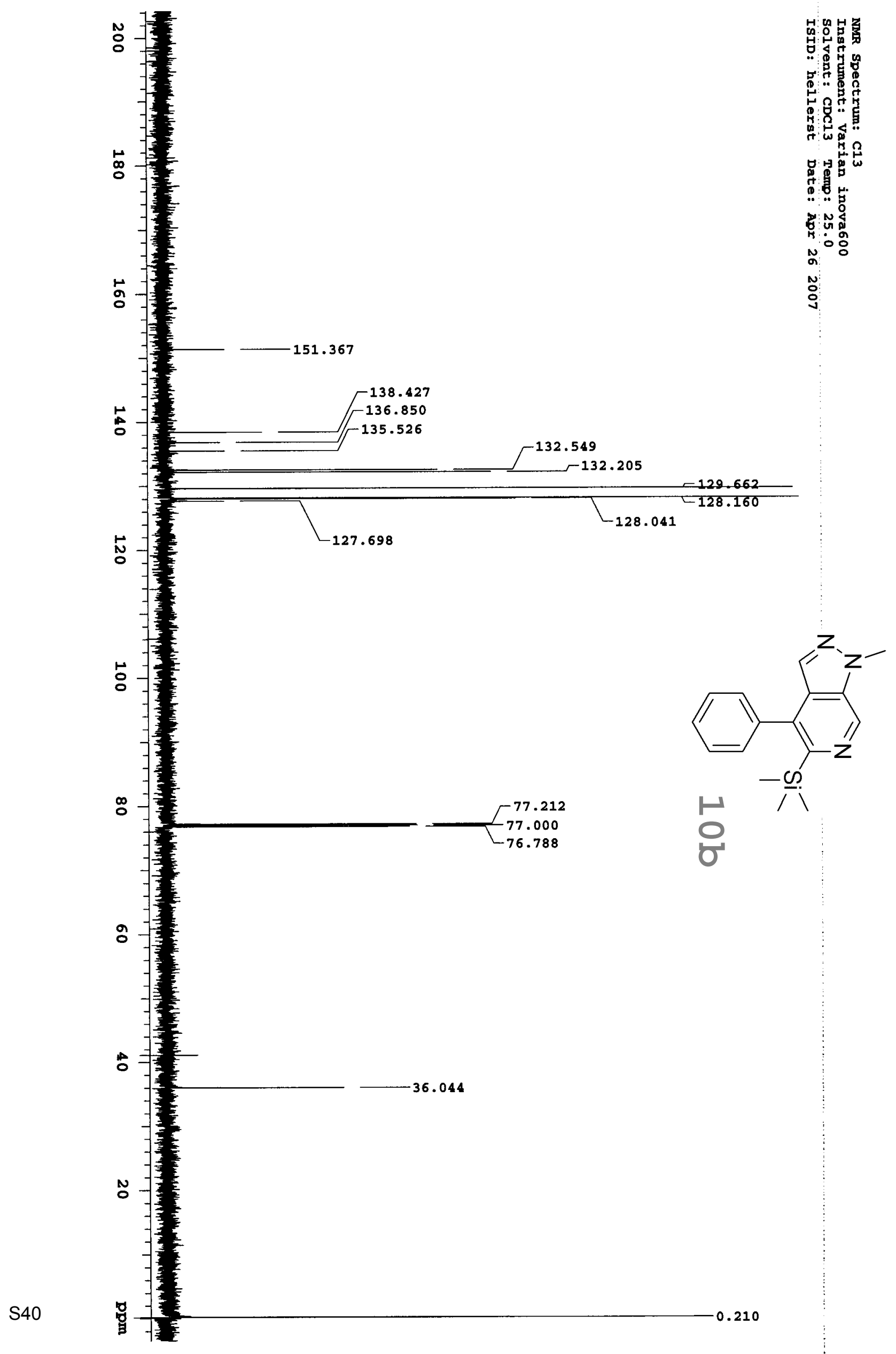




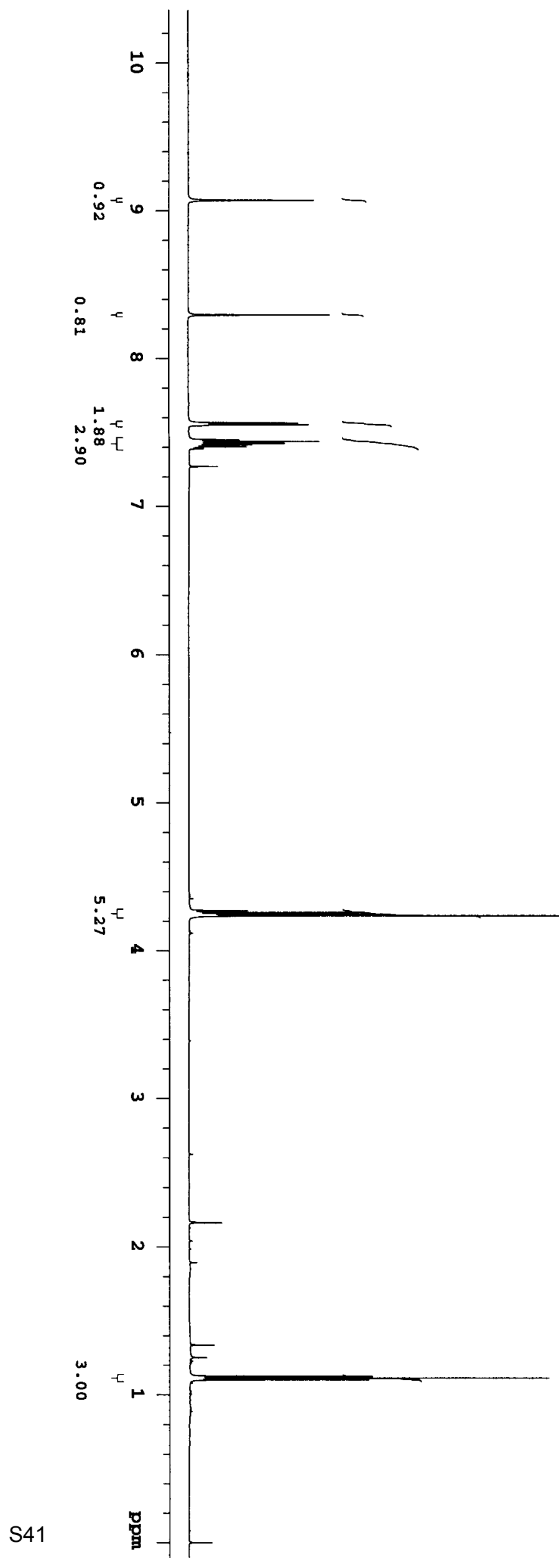

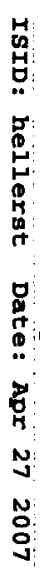

岁的

品

...

48.7

+ $w$

8

욤

氝

응

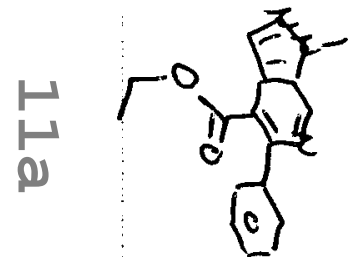




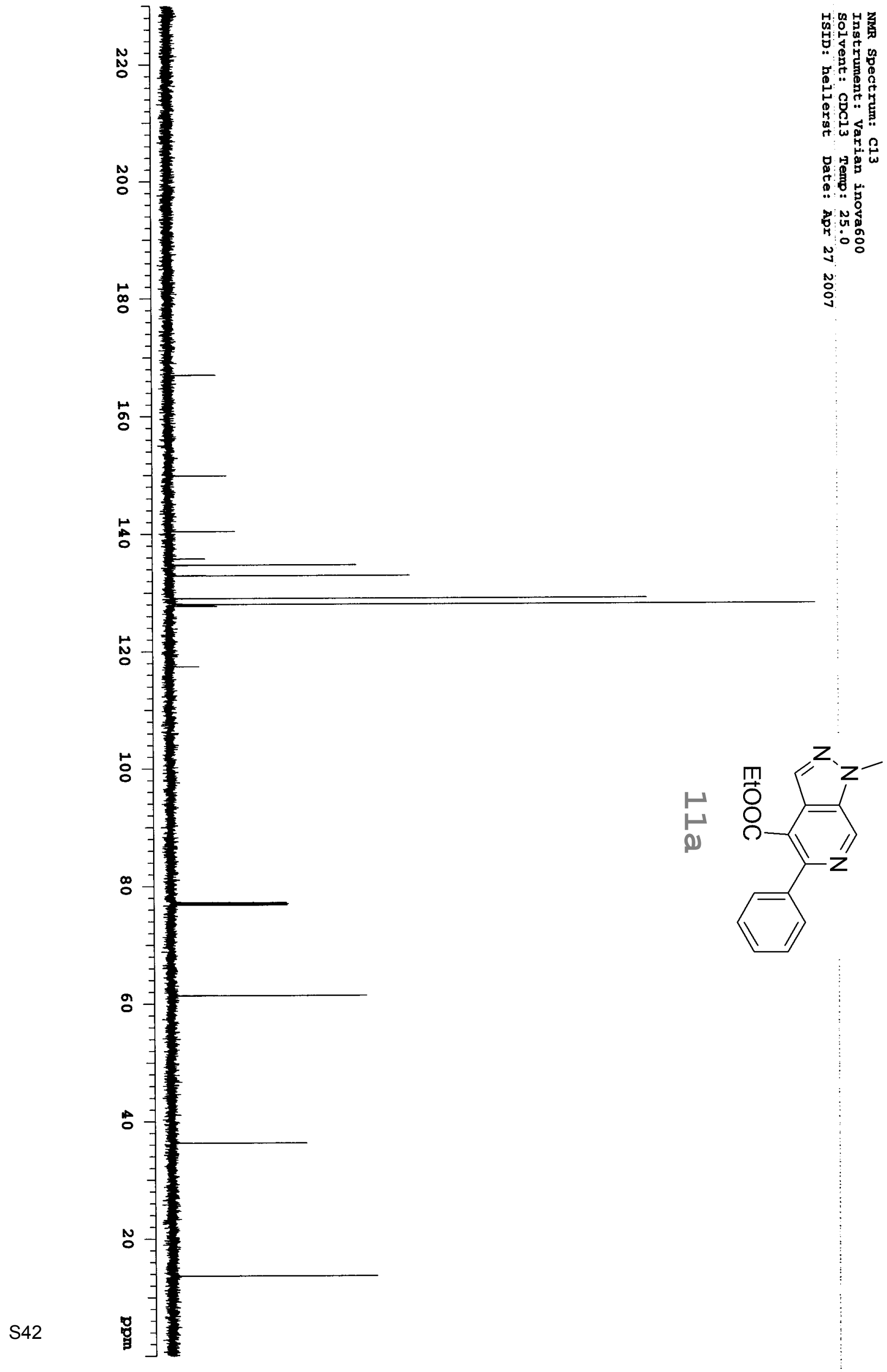




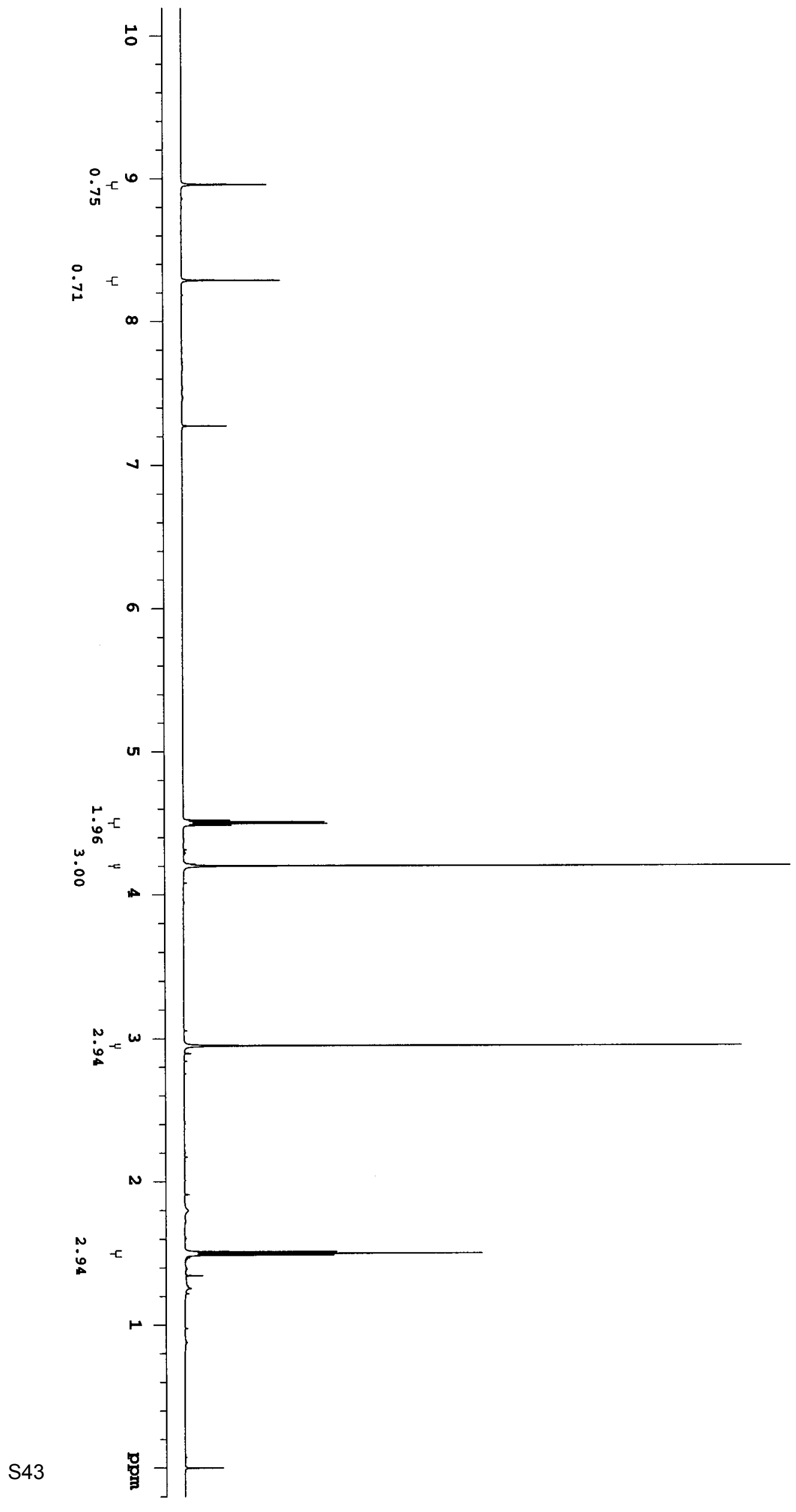

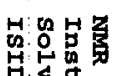

‥

प:

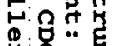

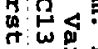

용

อ.

중

o:

:

v

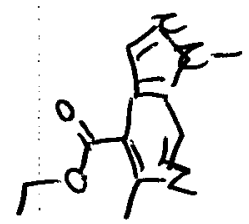



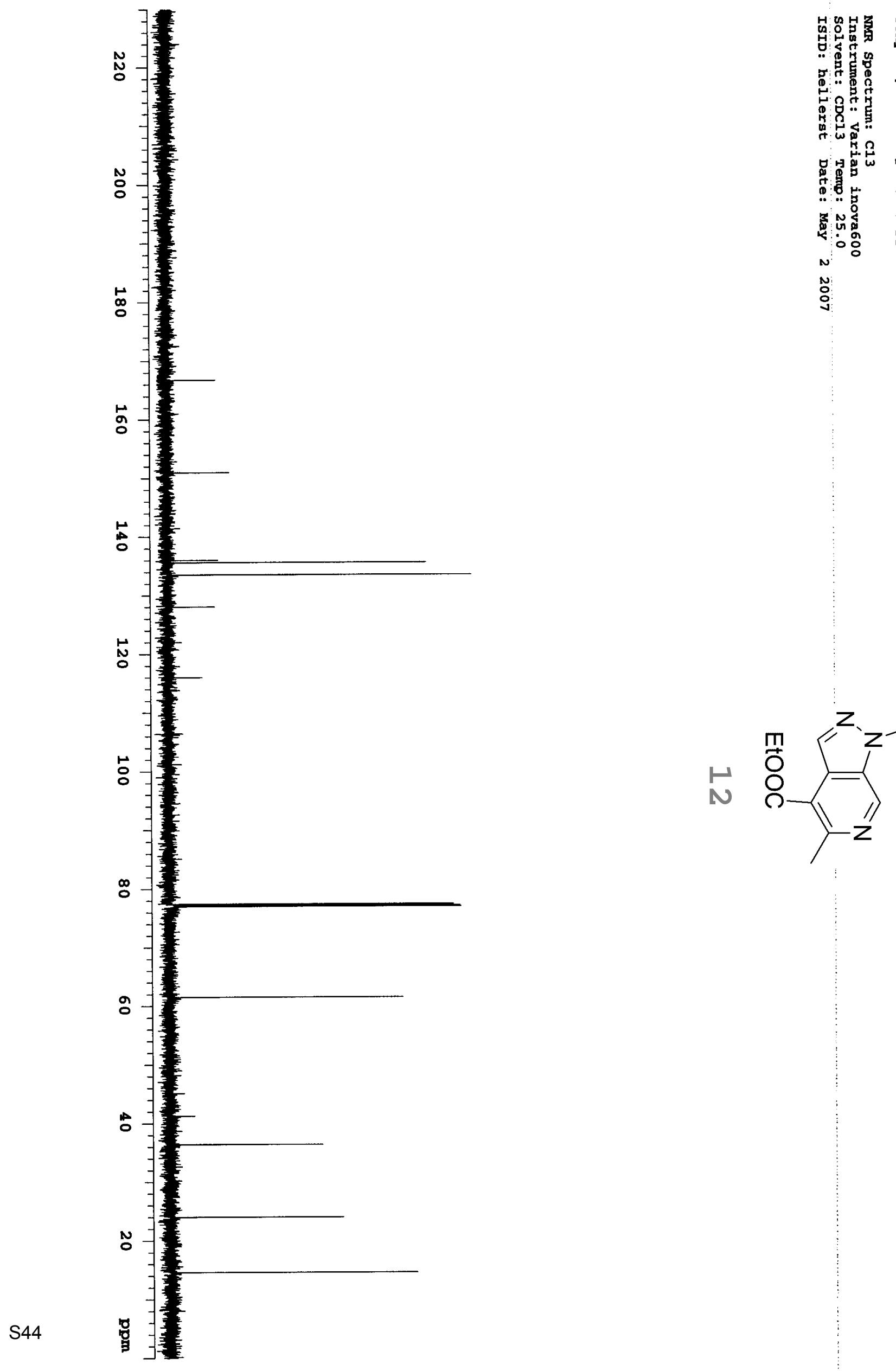$$
\text { 萿 }
$$$$
\text { i }
$$$$
\text { : }
$$$$
\text { 畩 }
$$$$
\text { 占 }
$$<smiles>C[Z]1ccc2c(O)c(C)[c]cc12</smiles> 


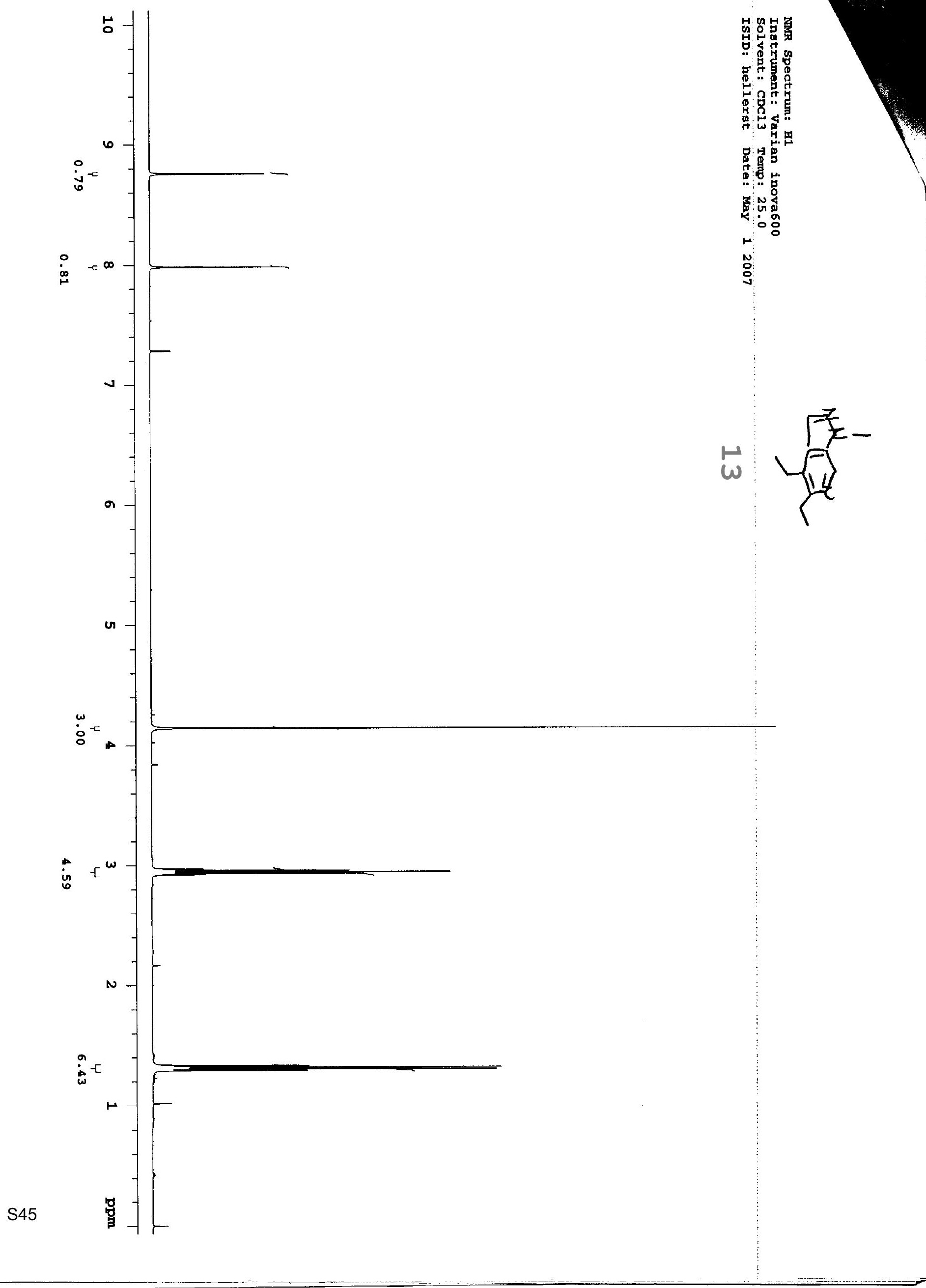




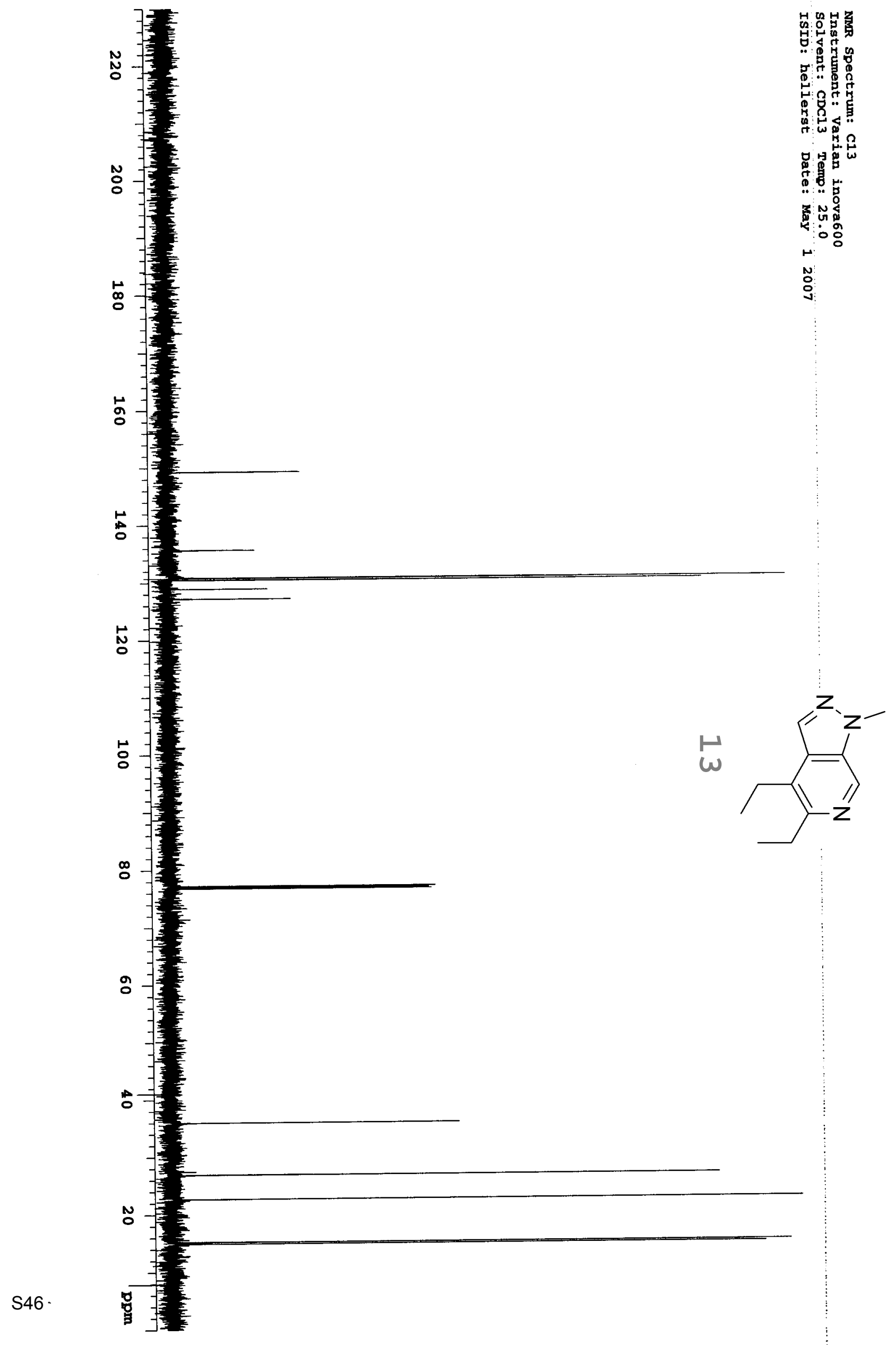




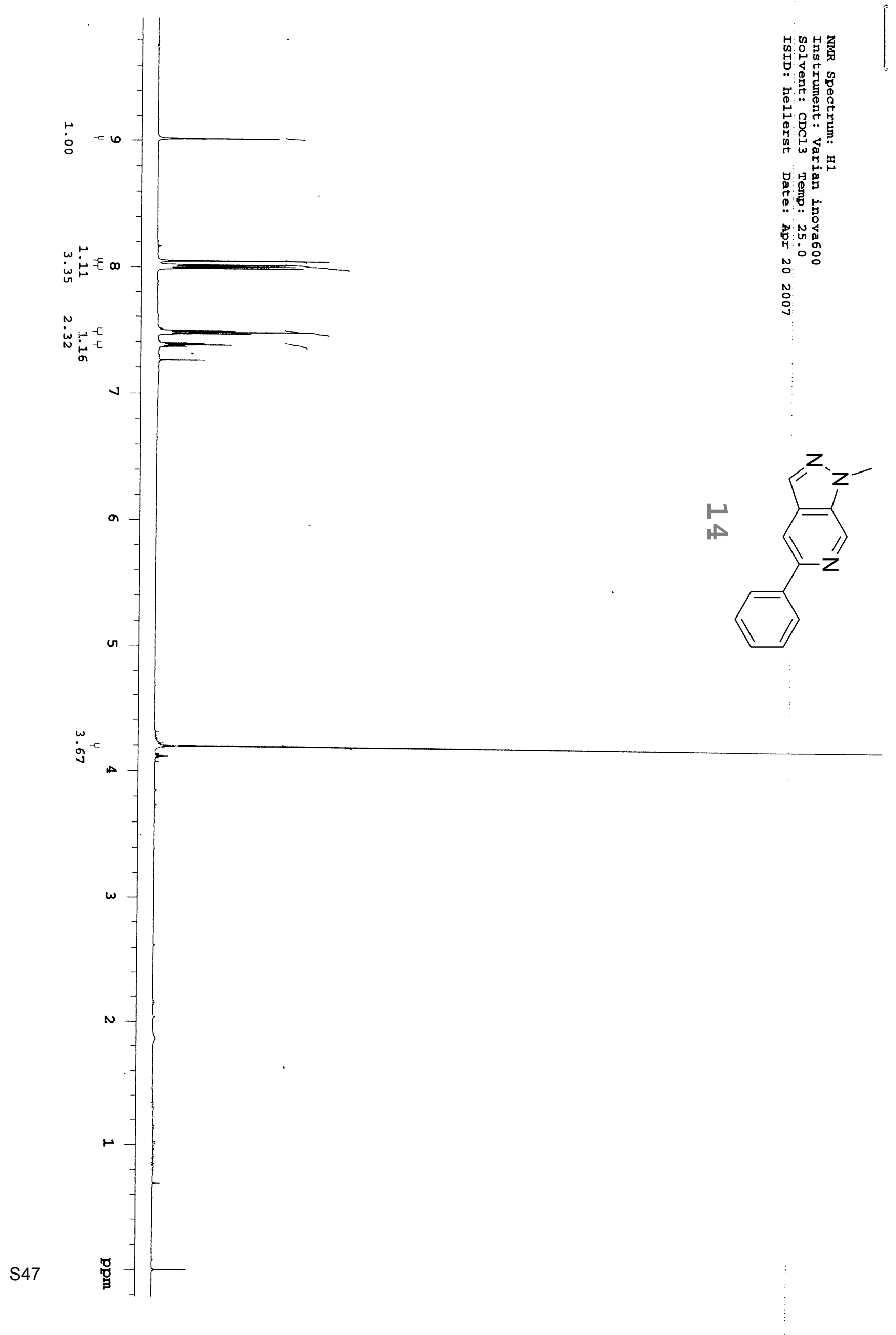




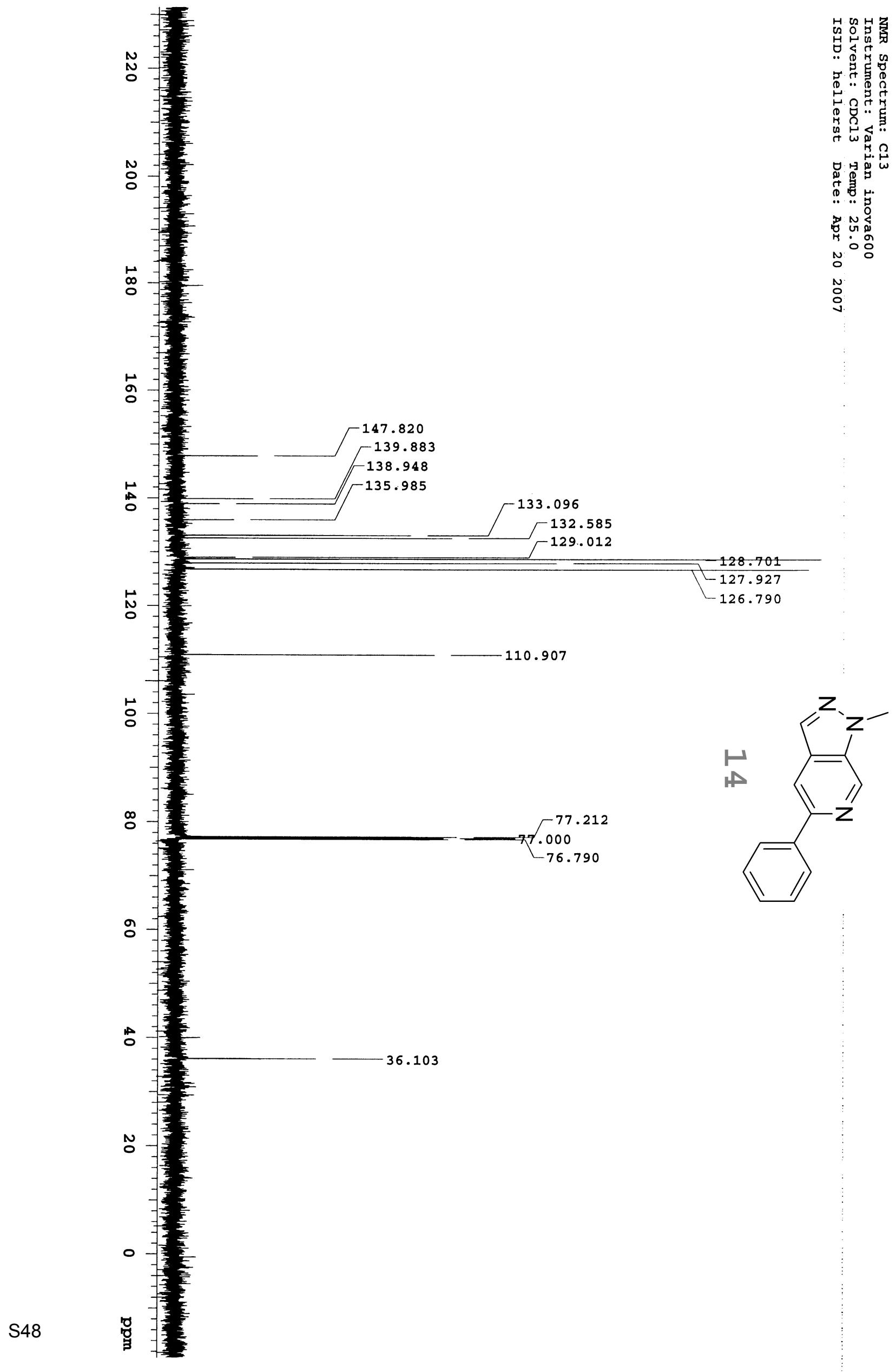



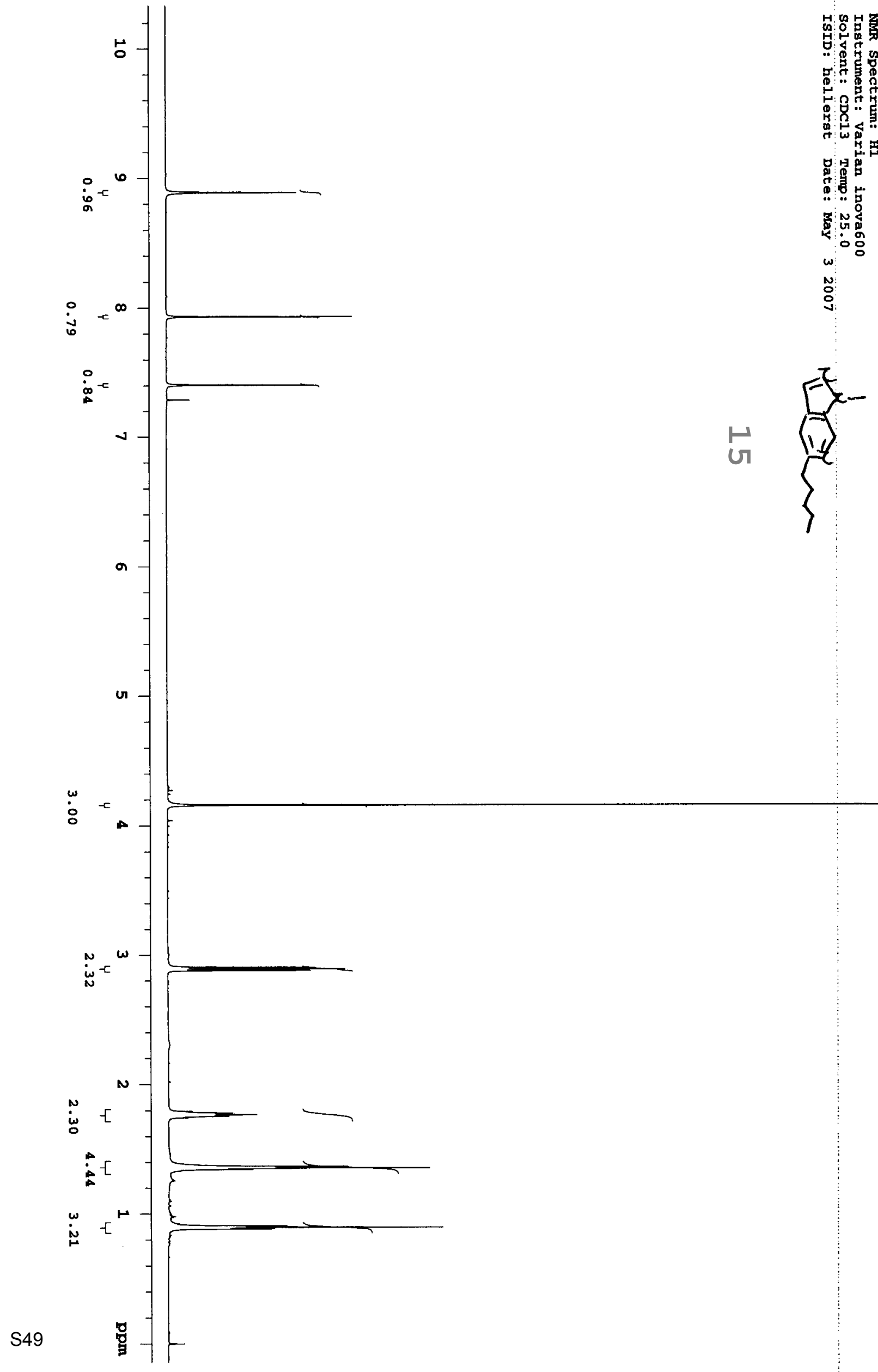


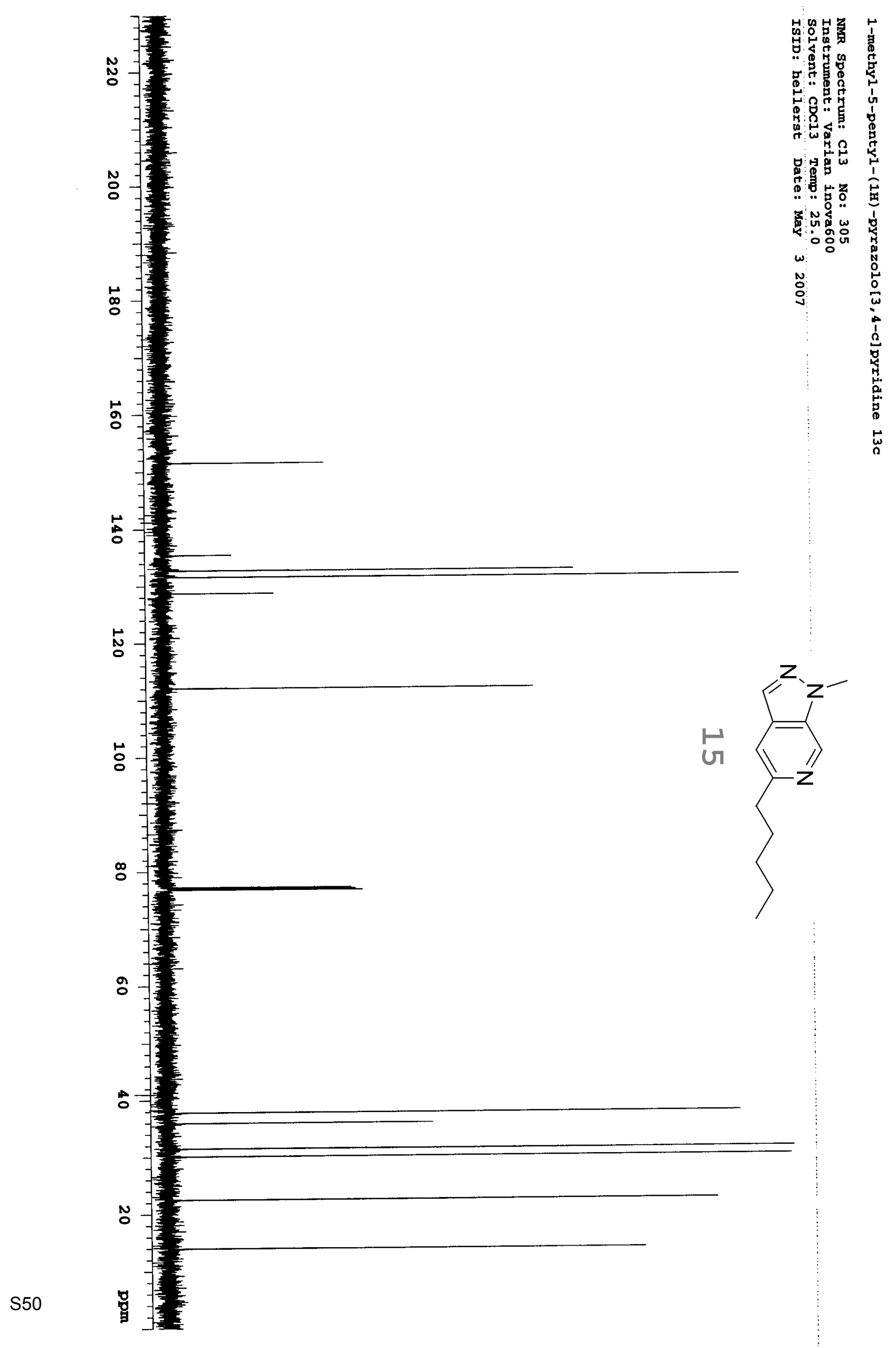




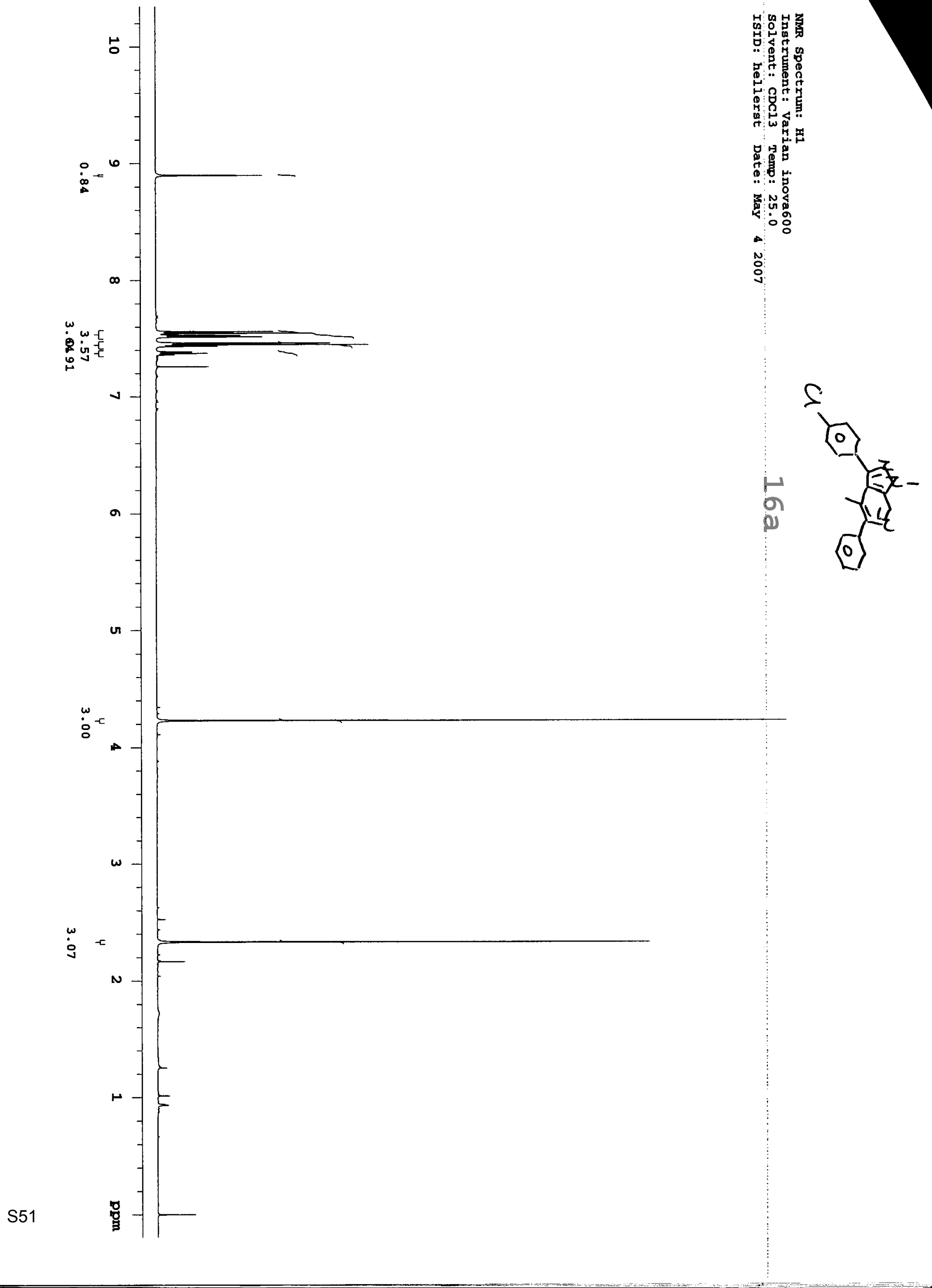




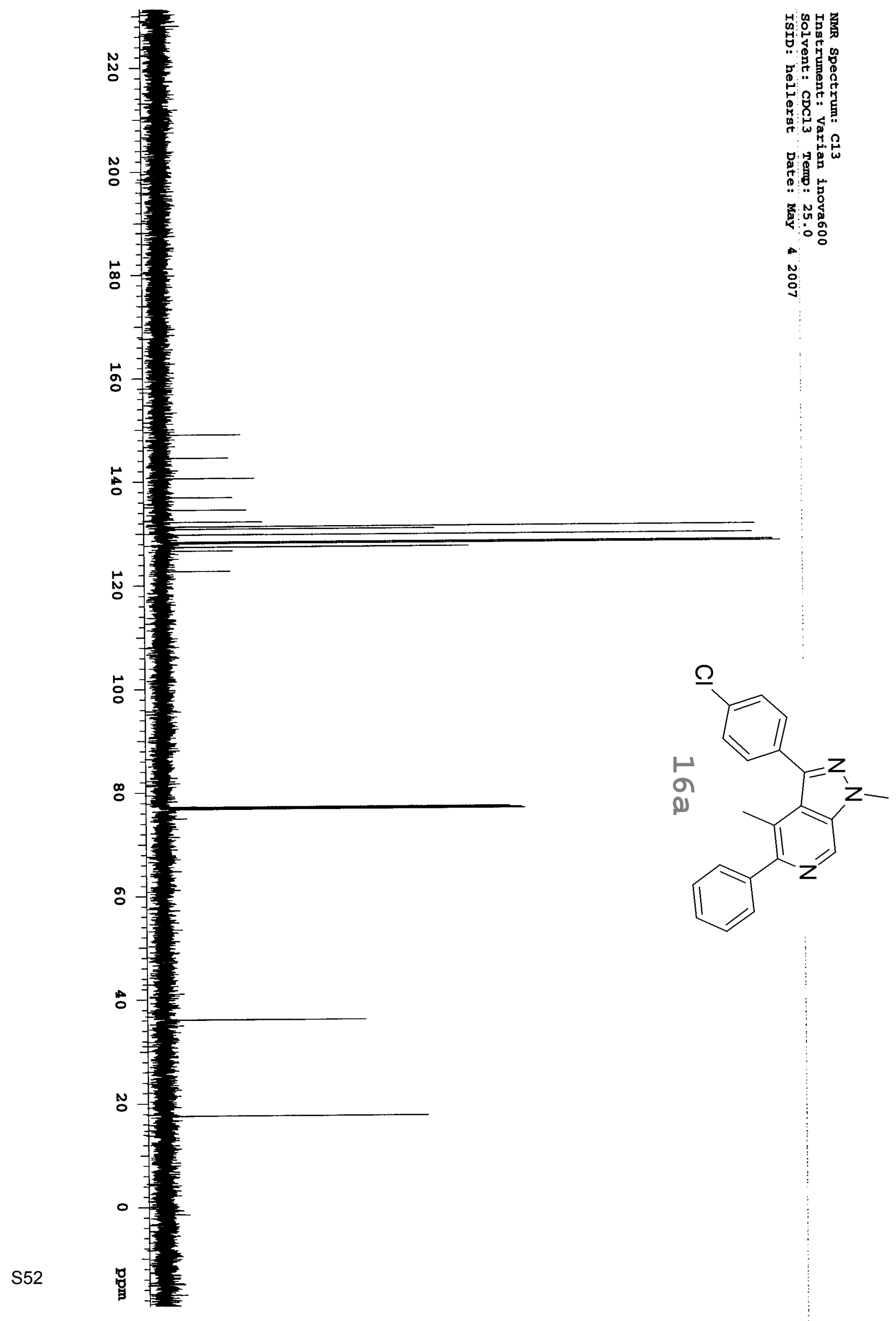



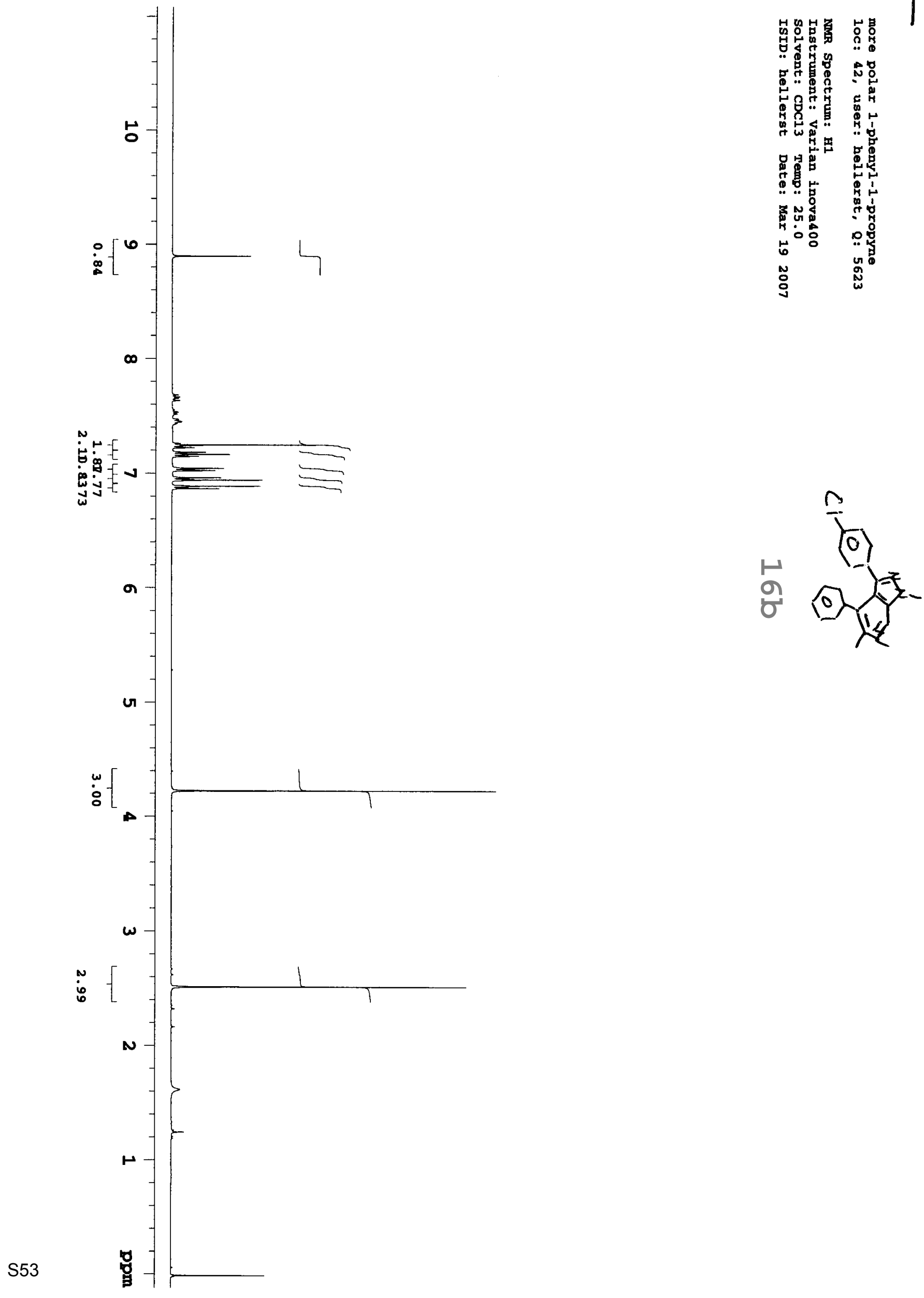


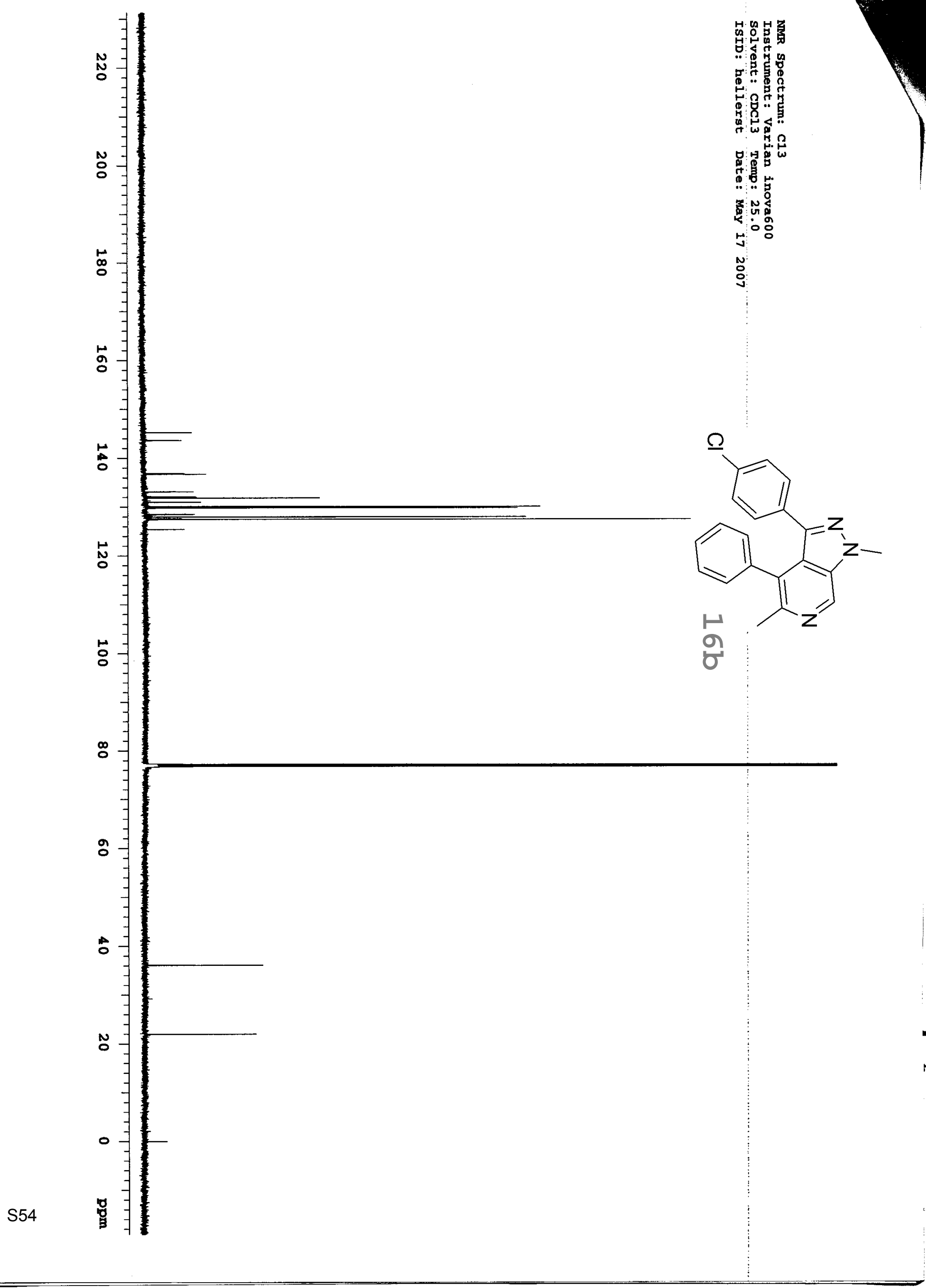




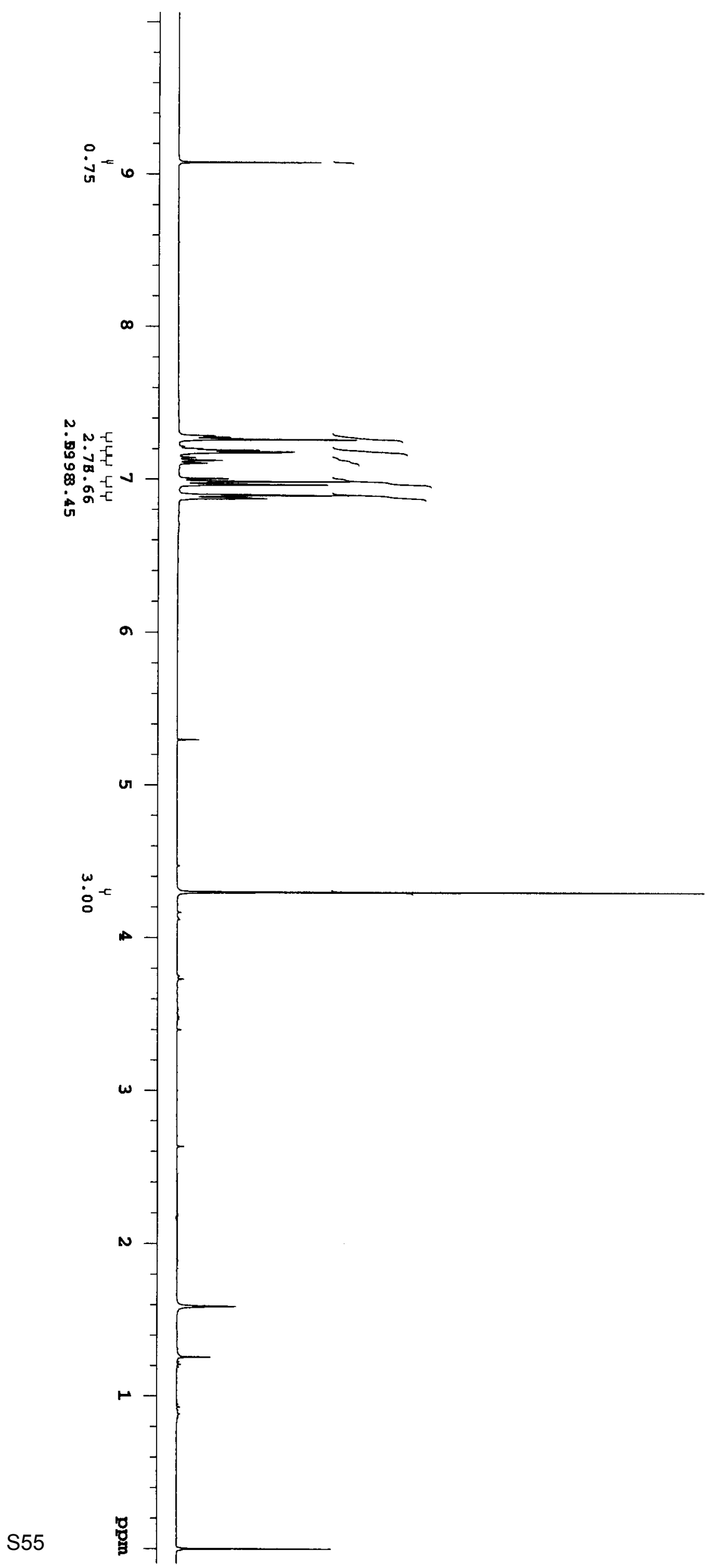

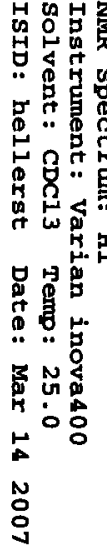

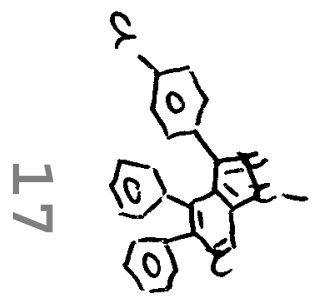




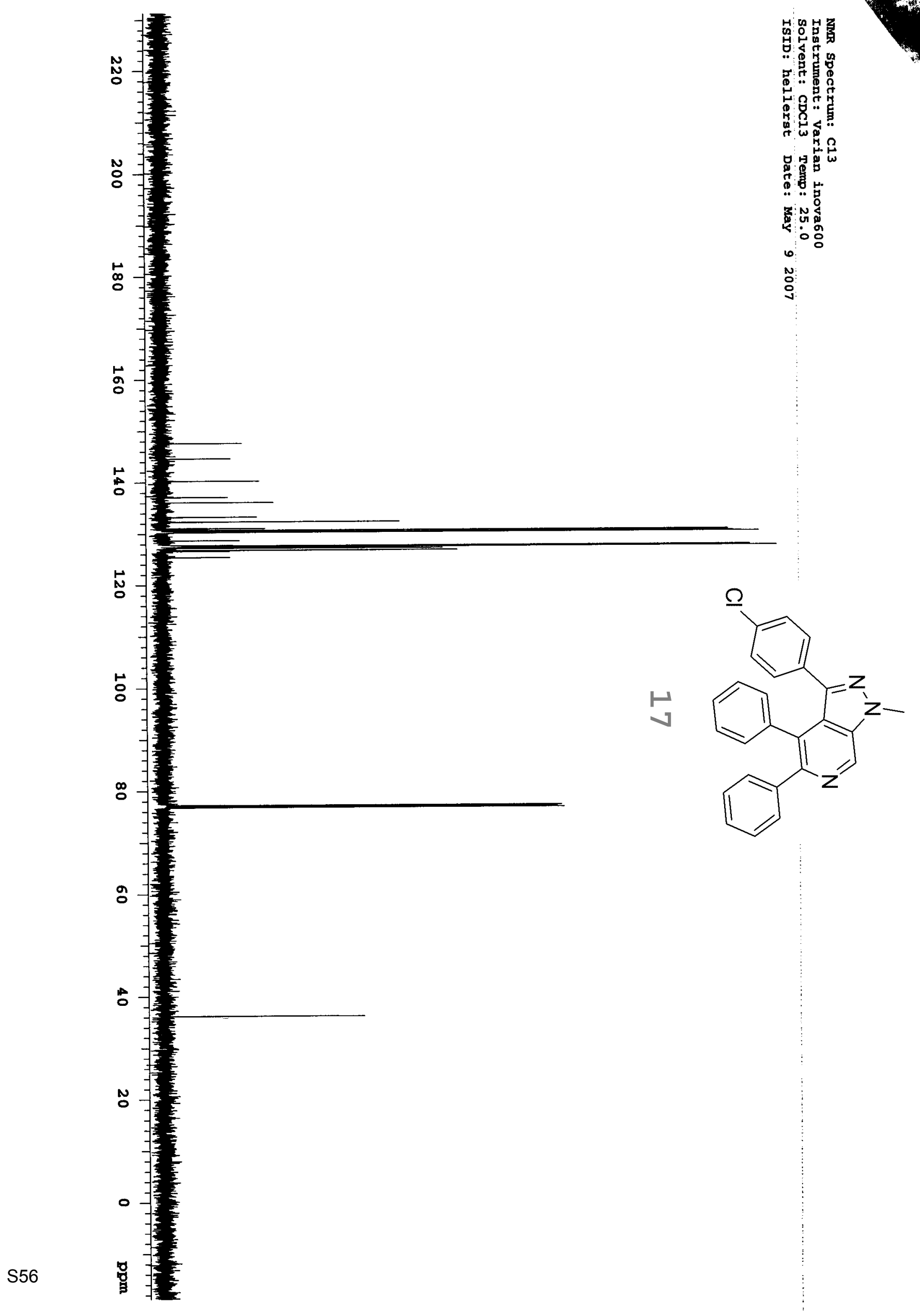




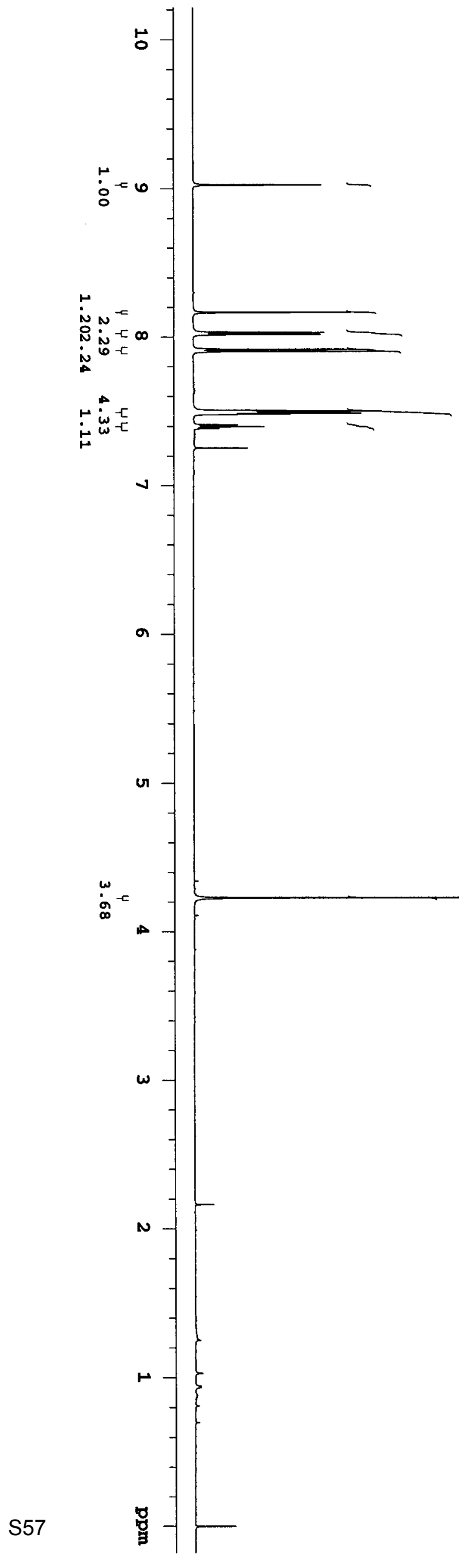

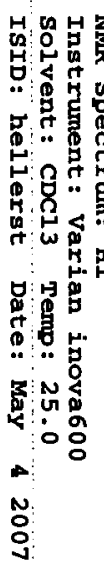

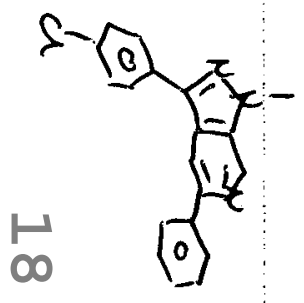




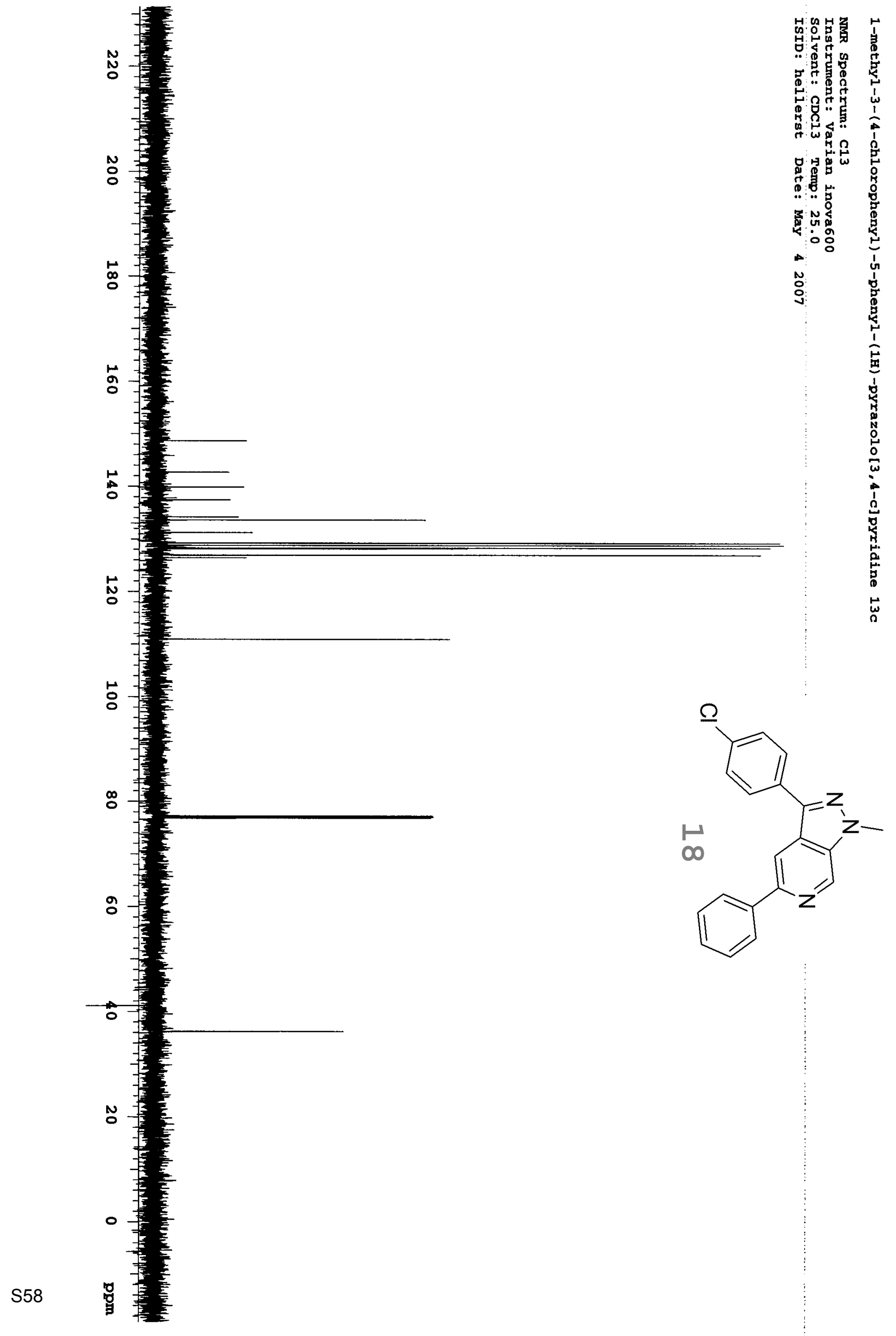




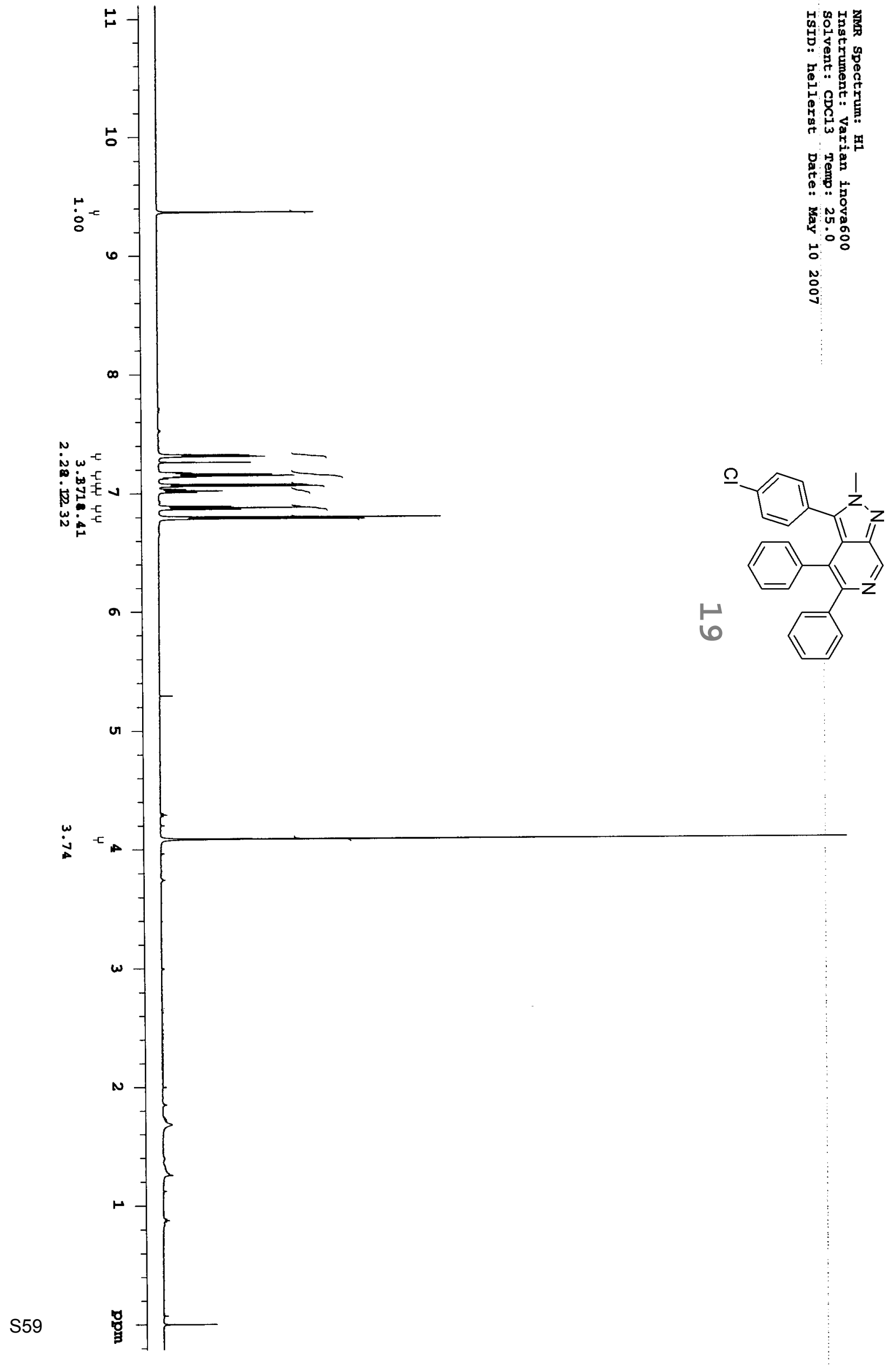




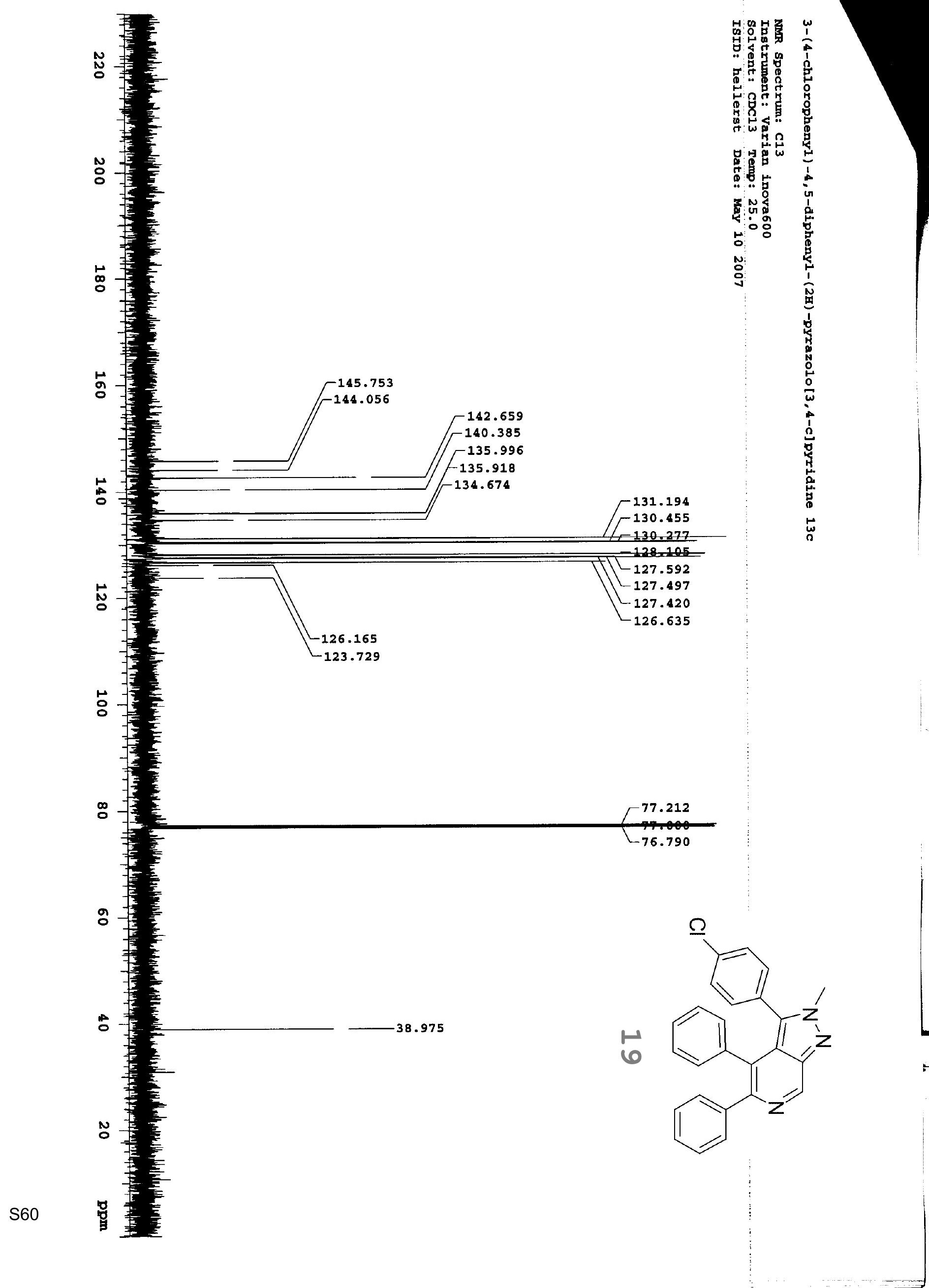

